\title{
Teashirt 3 Regulates Development of Neurons Involved in Both Respiratory Rhythm and Airflow Control
}

\author{
Xavier Caubit, ${ }^{1,4}$ Muriel Thoby-Brisson, ${ }^{2 \star}$ Nicolas Voituron, ${ }^{3 \star}$ Pierre Filippi, ${ }^{1 \star}$ Michelle Bévengut, ${ }^{3}$ Hervé Faralli, ${ }^{1}$ \\ Sébastien Zanella, ${ }^{3}$ Gilles Fortin, ${ }^{2}$ Gérard Hilaire, ${ }^{3}$ and Laurent Fasano ${ }^{1}$ \\ ${ }^{1}$ Unité Mixte de Recherche (UMR) 6216, Centre National de la Recherche Scientifique (CNRS), Université de la Méditerranée, Institut de Biologie du \\ Développement de Marseille Luminy, Parc Scientifique de Luminy, 13288 Marseille Cedex 9, France, ${ }^{2}$ Neurobiology and Development, Unité Propre de \\ Recherche 3294, Institute of Neurobiology A. Fessard, CNRS, 91198 Gif-sur-Yvette Cedex, France, ${ }^{3}$ MP3-Respiration, Centre de Recherche en \\ Neurobiologie et Neurophysiologie de Marseille, UMR 6231, CNRS, Université Aix Marseille II and III, Faculté Saint Jérôme, 13397 Marseille Cedex 20 , \\ France, and ${ }^{4}$ Université de Provence, 13331 Marseille, France
}

Neonatal breathing in mammals involves multiple neuronal circuits, but its genetic basis remains unclear. Mice deficient for the zinc finger protein Teashirt 3 (TSHZ3) fail to breathe and die at birth. Tshz3 is expressed in multiple areas of the brainstem involved in respiration, including the pre-Bötzinger complex (preBötC), the embryonic parafacial respiratory group (e-pF), and cranial motoneurons that control the upper airways. Tshz3 inactivation led to pronounced cell death of motoneurons in the nucleus ambiguus and induced strong alterations of rhythmogenesis in the e-pF oscillator. In contrast, the preBötC oscillator appeared to be unaffected. These deficits result in impaired upper airway function, abnormal central respiratory rhythm generation, and altered responses to $\mathrm{pH}$ changes. Thus, a single gene, Tshz3, controls the development of diverse components of the circuitry required for breathing.

\section{Introduction}

In mammals, breathing is a vital and complex motor act requiring rhythmic coordinated contractions of two sets of muscles, those governing the chest pump to induce airflow into and out of the lungs and those governing the upper airway valve to allow airflow. To secure blood oxygenation and survival at birth, the whole respiratory system must correctly mature during embryogenesis. This includes the brainstem respiratory rhythm generator (RRG) as well as motoneurons targeting chest muscles and those involved in airway opening. The RRG is composed of two interacting neuronal networks: the pre-Bötzinger complex (preBötC) (Smith et al., 1991) and the parafacial respiratory group (pFRG) (Onimaru and Homma, 2003). Required for survival at birth, the RRG is already functional at prenatal stages where it contributes to motor coordination of the respiratory apparatus and confers central chemosensitivity at embryonic stages (Thoby-Brisson and Greer, 2008; Dubreuil et al., 2009).

\footnotetext{
Received April 7, 2010; revised May 20, 2010; accepted June 3, 2010.

This work was supported by Centre Nationale de la Recherche Scientifique and by grants from the French Muscular Dystrophy Association (AFM). H.F. was supported by the AFM. We thank Q. Ma (Harvard Medical School, Boston, MA) for the vGlut2 probe, A. Garratt (Max Delbrück Center for Molecular Medicine, Berlin, Germany) for TSHZ3 antibody, and C. Goridis, J. F. Brunet (Ecole Normale Supérieure, Paris, France), and H. Enomoto (RIKEN Center for Developmental Biology, Hyogo, Japan) for PHOX2b antibodies. We acknowledge the contributions of F. Cayetanot, A.-M. Lajard, and C. Gestreau to some preliminary experiments and C. Henderson for critical reading of this manuscript.

${ }^{*}$ M.T.-B., N.V., and P.F. contributed equally to this work.

Correspondence should be addressed to Dr. Xavier Caubit, Institut de Biologie du Développement de Marseille Luminy, Unité Mixte de Recherche 6216, Centre National de la Recherche Scientifique, Parc Scientifique de Luminy, 13288 Marseille Cedex 9, France. E-mail: caubit@ibdm.univ-mrs.fr.

S. Zanella's present address: Seattle Children's Research Institute, Center for Neuroscience and University of Washington, Neurosurgery Department, Seattle, WA 98101.

DOI:10.1523/JNEUROSCI.1765-10.2010

Copyright $\odot 2010$ the authors $\quad 0270-6474 / 10 / 309465-12 \$ 15.00 / 0$
}

An important question in the neurobiology of breathing is to define the genetic mechanisms involved in the development and specification of central respiratory neuronal populations. Important progress has been made in the analysis of mechanisms required for generating diversity in brainstem motoneurons (Guthrie, 2007), but little is known about the genetic specification of the upper airway motoneurons. Moreover, although transcription factors such as MafB, Tlx1/3, Phox2b, Egr2, and Math1 have been shown to play important roles in the specification of neurons constituting the two oscillators of the RRG (Blanchi et al., 2003; Cheng et al., 2004; Pagliardini et al., 2008; Dubreuil et al., 2009; Rose et al., 2009; Thoby-Brisson et al., 2009), it is not clear how the necessary coordination with the airway motoneurons is set up. In addition, several of these mutations lead to absence of some of the neurons involved in respiratory control, meaning that the genetic control of functional rhythmogenesis has proved hard to decipher.

In Drosophila, the teashirt gene ( $t s h)$ encodes a zinc finger protein that is required to repress head developmental pathways and promote trunk identity (Fasano et al., 1991). Tsh-related genes (Tshz) have been characterized in vertebrates (Caubit et al., 2000, 2008; Manfroid et al., 2004; Koebernick et al., 2006; Coré et al., 2007; Onai et al., 2007; Kajiwara et al., 2009; Jenkins et al., 2010); however, their role in the development of the mammalian CNS is poorly understood. We previously reported that teashirt 3 (Tshz3) mutant mice develop to term but fail to breathe and die quickly at birth (Caubit et al., 2008), suggesting that Tshz3 might play a role in the development of the peripheral and/or central control of respiration. Here, we investigated the neuronal mechanisms underlying the premature death of these mice using a multidisciplinary approach. 
We report that $T s h z 3$ inactivation specifically leads to death of nucleus ambiguus (nA) motoneurons governing the upper airways and prevents the functional emergence of the embryonic pFRG (e$\mathrm{pF}$ ), leading to abnormal RRG function and loss of respiratory responses to $\mathrm{pH}$ challenges. Our results provide the first evidence for a critical function of $T s h z 3$ in both promoting survival of nA motoneurons, which govern upper airway function, and establishing a RRG activity compatible with survival at birth.

\section{Materials and Methods}

Mouse lines

All the mouse lines used in this study were maintained on a CD1 background. The $T s h z 3^{+/ l a c Z}$ allele carries an in-frame insertion of lac $Z$ coding sequence within the second exon of Tshz3 (Caubit et al., 2008). All experiments were performed in accordance with national (JO 87-848) and European legislation (86/609/CEE) on animal experimentation.

\section{Histology, $\beta$-galactosidase staining, immunohistochemistry, immunofluorescence, and in situ hybridization}

Nissl stain and $\beta$-galactosidase ( $\beta$-gal) histochemistry were done as described previously (Caubit et al., 2005, 2008). For immunodetection, brainstems were dissected and fixed for $2 \mathrm{~h}$ in $4 \%$ paraformaldehyde (PFA)/PBS, cryoprotected overnight in PBS/20\% sucrose, embedded in OCT, and sectioned at 16 or $20 \mu \mathrm{m}$. Sections were incubated overnight at $4^{\circ} \mathrm{C}$ with primary antibodies. The following antibodies were used: guinea pig and rabbit anti-TSHZ3 antibodies (1/2000; gift from A. Garratt, Max Delbrück Center for Molecular Medicine, Berlin, Germany) (Caubit et al., 2008); rabbit anti-NK1R (1/5000; Sigma-Aldrich); rabbit antisomatostatin (1/500; Bachem/Peninsula Laboratories); mouse antiISLET1/2 (2D6, 1/500; 4D5, 1/100; Developmental Studies Hybridoma Bank); rabbit anti-PHOX2b (1/1000; gift from J. F. Brunet and C. Goridis, Ecole Normale Supérieure, Paris, France); guinea pig anti-PHOX2b (1/1000; gift from H. Enomoto, RIKEN Center for Developmental Biology, Hyogo, Japan); rabbit anti-5-HT (1/5000; Sigma-Aldrich); and rabbit anti-activated caspase-3 (1/100; Cell Signaling). For analysis of innervations and synaptic sites in diaphragms, we used the mouse antineurofilament (2H3; 1/2500; Developmental Studies Hybridoma Bank) and Alexa Fluor 488-conjugated $\alpha$-bungarotoxin (1/2000; Invitrogen). Primary antibodies were revealed with secondary antibodies coupled to Alexa 488, 546 (1/1000), and Cy5 (1/500) (Invitrogen). Immunostained sections were examined and processed using Zeiss $100 \mathrm{M}$ confocal microscope, LSM510 META, and LSM510 software. All figures were color corrected and assembled using Adobe Photoshop.

The number of neurons in the $\mathrm{nA}$ was evaluated by counting PHOX2b + cells on $20 \mu \mathrm{m}$ consecutive sagittal sections for six distinct embryos of each genotype at embryonic day 15.5 (E15.5). The number of activated caspase- $3+$ cells was evaluated on sagittal sections in the PHOX $2 \mathrm{~b}+$ cell population extending caudally to the dorsal part of the facial nucleus (nVII) over a rostrocaudal distance of $350 \mu \mathrm{m}$. Apoptotic cells were detected using the apopTag In Situ Apoptosis Detection kit (Millipore Bioscience Research Reagents). Counts of e-pF cells were made within an area delimited ventrally by the medullary surface, dorsally by the nVII, and extending from the rostral end of the nVII to 200 $\mu \mathrm{m}$ caudal to the nVII. Cells were counted at E15.5 on all consecutive sagittal sections and/or on every other transverse section for six wild-type (WT) and six mutants. The number of medullary 5-HT neurons was estimated by counting 5-HT-positive cells on every fourth coronal sections throughout the caudal serotoninergic cluster.

Whole-mount neurofilament stainings were performed as described previously (Maina et al., 1997). In situ hybridization on cryosections or on whole-mount preparations of embryos was performed as described previously (Tiveron et al., 1996; Hirsch et al., 1998). Antisense RNA probes for peripherin (Escurat et al., 1990), Tshz3 (Caubit et al., 2005), and Vglut2 (Cheng et al., 2004) were labeled using a DIG-RNA labeling kit (Roche). Atoh1 cDNA probe was from V. Dubreuil (Ecole Normale Supérieure, Paris, France).
In vivo breathing studies

As reported previously (Blanchi et al., 2003; Viemari et al., 2005), breathing movements of surgically delivered E18.5 embryos were recorded for 3-5 min by the whole-body plethysmography technique in thermostated chambers equipped with a differential pressure transducer (EMKA Technologies). Mouth temperature was monitored with a miniature thermistor nylon-coated probe. Chest muscles electromyograms and cardiac pulses were recorded with two thin insulated copper wires $(100 \mu \mathrm{m}$ diameters) slightly inserted through the skin in the lateral parts of the caudal rib cage. Similarly, chest muscle electromyograms were occasionally recorded in vitro when chest movements were observed during the dissection of en bloc preparations at E18.5. For in utero recordings, three pregnant mice were deeply anesthetized with pentobarbital (60 $\mathrm{mg} \cdot \mathrm{kg}^{-1}$ ), a median incision was made along the white line to access the uterine horns and a given embryo was isolated, with preserved umbilical irrigation, and thin cooper wires were inserted in chest muscle as reported above. Electromyographic and cardiac signals were filtered, amplified (Neurolog System; Digitimer), and visualized on memory scope or stored on PC.

\section{In vitro preparations}

Isolated hindbrain and transverse slice preparations were obtained as previously described (Thoby-Brisson et al., 2005, 2009). Briefly, pregnant mice were killed by cervical dislocation at the desired developmental stage from E14.5 up to E18.5. Embryos were removed from the uterine horns and placed in oxygenated artificial CSF (aCSF) with the following composition (in mM): $120 \mathrm{NaCl}, 8 \mathrm{KCl}, 1.26 \mathrm{CaCl}_{2}, 1.5 \mathrm{MgCl}_{2}, 21$ $\mathrm{NaHCO}_{3}, 0.58 \mathrm{Na}_{2} \mathrm{HPO}_{4}, 30$ glucose, $\mathrm{pH} 7.4$, and was equilibrated with carbogene $\left(95 \% \mathrm{O}_{2}-5 \% \mathrm{CO}_{2}\right)$. Acidic aCSF, $\mathrm{pH} 7.2$, was obtained by reducing by one-half the $\mathrm{NaHCO}_{3}$ concentration, and osmolarity was compensated by addition of equivalent $\mathrm{NaCl}$. Embryos were kept in aCSF at room temperature until used in electrophysiological and optical recording sessions. Brainstems and slices were dissected in aCSF at $4^{\circ} \mathrm{C}$. En bloc brainstem preparations were isolated by an anterior section performed at the level of the isthmus and a posterior section performed caudal to the fourth cervical roots (C4). Isolated brainstem preparations were then transferred into the recording chamber ventral side up. Transverse preBötC slice preparations were obtained by sectioning isolated brainstem preparations embedded in an agar block using a vibratome (Leica VT1000s; Leica). Serial sections were performed from rostral to caudal until the posterior limit of the nVII (visible in direct light) was reached. Then, $200 \mu \mathrm{m}$ more posteriorly, a $450-\mu \mathrm{m}$-thick slice containing the preBötC at its anterior side was obtained and placed into the recording chamber with this rostral surface up.

\section{Electrophysiology}

All recordings were done blind, the genotype of the embryos being unknown at the time of the experiments.

Motor nerve activity. Motor nerve activity was recorded from the fourth cervical root using a suction electrode (with a $100 \mu \mathrm{m}$ tip diameter), fabricated from a filamented borosilicate glass tube (Clark GC 120F; Harvard Apparatus). The micropipette was filled with aCSF and connected to a high-gain AC amplifier (7P511; Grass Instruments) through silver wires. Local population activities (e-pF and preBötC oscillators) were recorded using the same method. The collected signal was filtered (bandwidth, $3 \mathrm{~Hz}$ to $3 \mathrm{kHz}$ ), rectified, and integrated using an electronic filter (time constant, $100 \mathrm{~ms}$; Neurolog System).

Patch-clamp recording. Whole-cell patch-clamp recordings of e-pF neurons were performed under visual control using differential interference contrast and infrared video microscopy, and using an Axoclamp 2A amplifier (Molecular Devices). All electrophysiological signals were stored on a computer via a digitizing interface (Digidata 1322A; Molecular Devices) and analyzed with the Pclamp9 software (Molecular Devices).

Recorded neurons were selected by their position (ventrolateral to the nVII) and their discharge pattern compared with the e-pF population activity. Patch electrodes were pulled from borosilicate glass tubes (Clark GC 150TF; Harvard Apparatus) and filled with a solution containing the following (in mM): $140 \mathrm{~K}$-gluconic acid, $1 \mathrm{CaCl}_{2} \cdot 6 \mathrm{H}_{2} \mathrm{O}, 10$ EGTA, 2 $\mathrm{MgCl}_{2}, 4 \mathrm{Na}_{2} \mathrm{ATP}, 10$ HEPES, pH 7.2, added with $1 \mathrm{mg} / \mathrm{ml}$ biocytin 

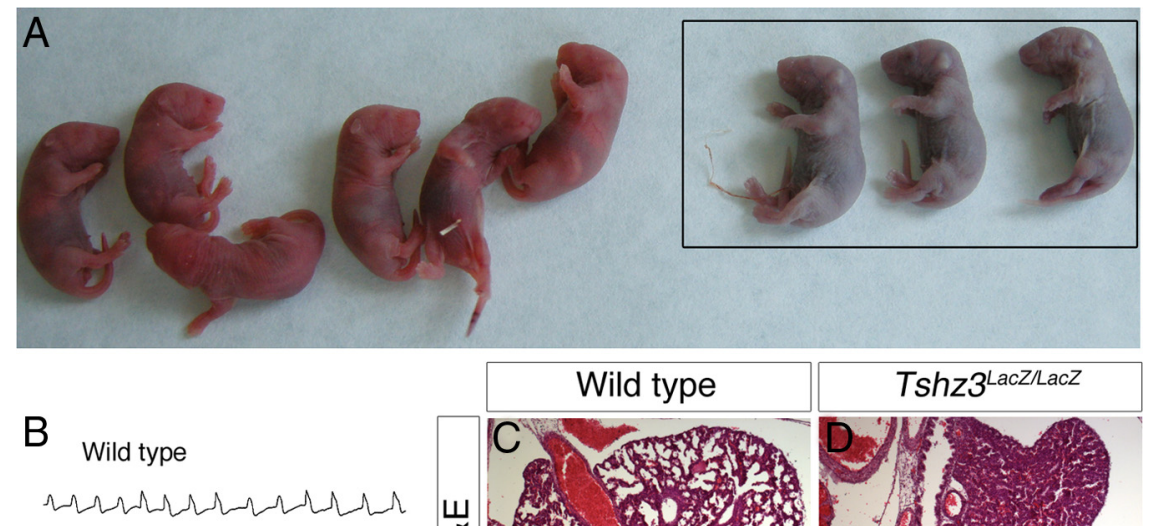

Tshz'LacZlLacZ
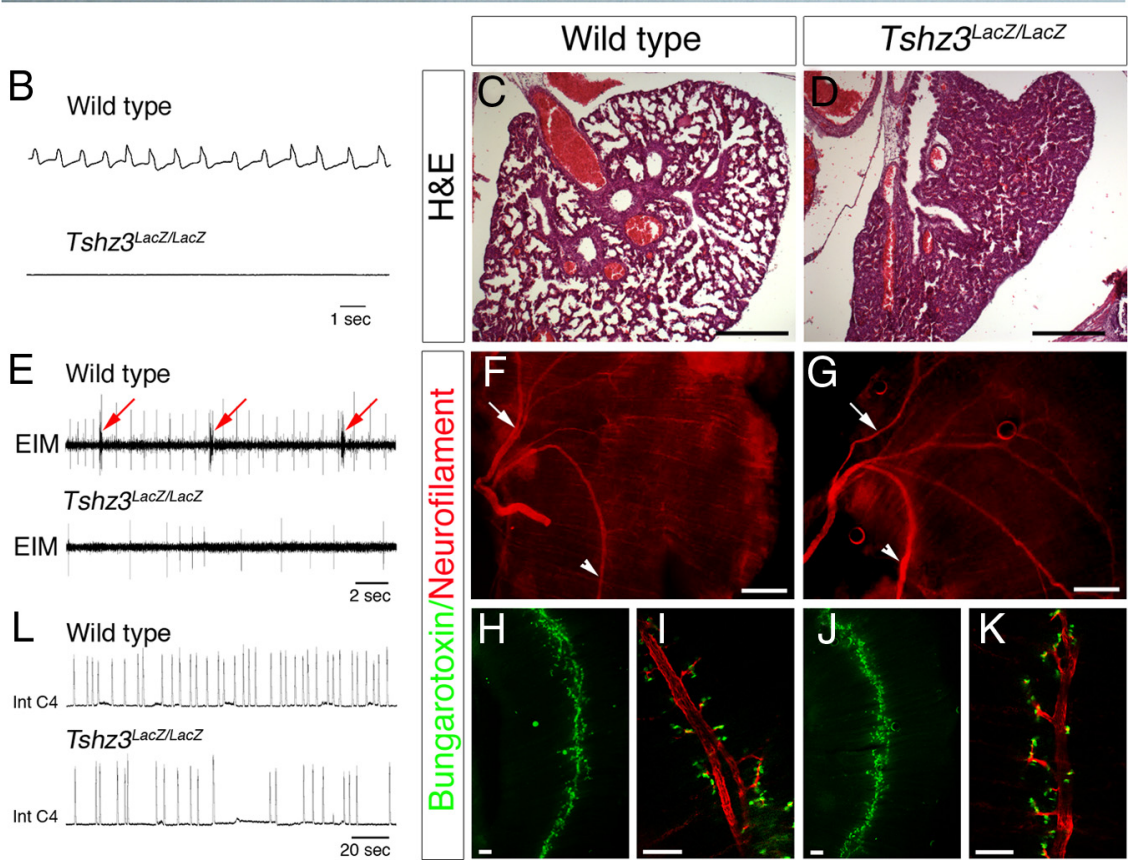

Figure 1. Tshz3 mutants do not breathe in vivo and generate slower centrally generated respiratory-like rhythm in vitro.A, Appearance of newborn immediately after birth. The Tshz3 homozygous mutants showed marked cyanosis (framed). $\boldsymbol{B}$, Plethysmographic recordings

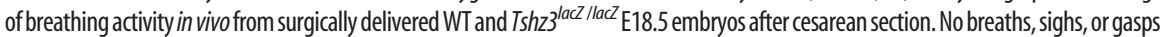
were detected in $T$ shz $3^{\text {lac I IlacZ }}$, whereas they commonly occurred in WT. C, D, Hematoxylin/eosin staining of transverse sections of control and $T s h z 3$ mutant lungs at $\mathrm{PO}$. Control display normal alveolar appearance. Newborn mutant lung alveoli were considerably reduced in size,

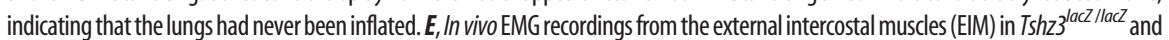
WT at E18.5 showing contractions in WT (arrow) but not in $T$ shzz $3^{\text {lac Ilac. }} . \boldsymbol{F}, \mathbf{G}$, Neurofilament staining on diaphragms from WT $(\boldsymbol{F})$ and $T_{\text {Ishzzlac Illacz }}(\boldsymbol{G})$ embryos. The arrows and arrowheads indicate sternal branches and crural branches of the phrenic nerve, respectively. Dorsal side of the diaphragm is up. Diaphragms from WT $(\boldsymbol{H})$ and $T s h z 3^{\text {lac Illac }}(\boldsymbol{J})$ embryos were stained for acetylcholine receptors (AchRs). As in WT embryos, an AChR clustering band was present at the center of muscle in the mutant. $\boldsymbol{I}, \boldsymbol{K}$, High magnification of branches of the phrenic nerve (red) showing AChRs (green). $L$, Integrate phrenic burst discharge recorded in vitro in medullary-spinal cord preparations

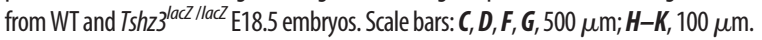

(Invitrogen). The pipette resistance was 5-6 M $\Omega$ when filled with this solution.

After the recording session, the patch pipette was carefully removed to keep the anatomical integrity of the neuron and the preparation was fixed in $4 \%$ PFA for $2 \mathrm{~h}$. The biocytin-filled recorded neurons were later revealed using Extravidine-FITC (1:400; Sigma-Aldrich) and their immunoreactivity for PHOX2b (see above) and, in the Tshz $3^{\text {lacz/lac }}$ mice, for $\beta$-gal (1:1000; Abcam), tested with a secondary antibody coupled to Alexa647 (Invitrogen).

\section{Calcium imaging}

Preparations (isolated hindbrains and transverse slices) were incubated at room temperature for $40 \mathrm{~min}$ in oxygenated aCSF containing the cell-permeant calcium indicator dye Calcium Green-1 AM (10 $\mu \mathrm{M}$; Invitrogen) diluted in DMSO $(10 \mu \mathrm{M})$. After incubation, preparations were transferred into the recording chamber, ventral side up and rostral side up for whole hindbrain and slices, respectively, and perfused continuously with oxygenated aCSF at $30^{\circ} \mathrm{C}$ for a $30 \mathrm{~min}$ recovery period allowing wash out of the dye excess. Optical recordings were performed using an upright epifluorescent microscope (E-600-FN; Nikon), equipped with a fluorescein filter block and a cooled CCD camera (Coolsnap HQ; Photometrics). Images were continuously captured using an exposure time of $100 \mathrm{~ms}$ in simultaneous exposure and readout mode. Optical images were analyzed using the MetaMorph software (Molecular Devices). The average changes in fluorescence in the areas of interest were calculated for each frame. Changes in fluorescence are expressed as the ratio of change relative to the initial value of fluorescence $(\Delta F / F)$.

\section{Data expression and statistics}

In vivo respiratory frequency and heart rate were expressed in cycles per minute $\left(\mathrm{c} \cdot \mathrm{min}^{-1}\right)$. In vitro phrenic nerve, preBötC, and e-pF neuronal population activity frequencies were expressed in burst/minute. The burst duration of $\mathrm{e}-\mathrm{pF}$ neurons was assessed by measuring the delay between the ascending and the descending phases of individual bursts when the membrane potential reached $-30 \mathrm{mV}$, a value at which the burst is fully developed. To compare mean frequencies between WT and Tshz $3^{\text {lac Z /lacZ }}$ mice or between two different experimental conditions, statistical analysis was done using Student's $t$ test. To compare cell counts, statistical analysis was done by using a two-tailed $t$ test or Mann-Whitney and StatEL software. All values are given as mean \pm SEM, and differences were assumed to be statistically different at $p<0.05$.

\section{Results}

$T s h z 3$ inactivation leads to haploinsufficiency, neonatal lethality, and dysfunction of central respiratory networks

The mouse $T s h z 3$ gene was inactivated as previously described (Caubit et al., 2008). One-half of the heterozygote progeny $T s h z 3^{+/ l a c Z}$ generated by crossing chimeric male founders and CD1 females died shortly after birth. Surviving $T s h z 3^{+/ l a c} Z$ pups were smaller than their WT siblings but recovered normal growth at $6-8$ weeks, and adults were healthy and fertile. Homozygous mutant embryos (Tshz $3^{\text {lacZ/lac }}$ ) developed at the expected Mendelian ratio and showed no external anatomical differences compared with WT littermates. At birth, however, Tshz $3^{\text {lac } Z / l a c Z}$ neonates failed to produce the first breath, quickly became cyanotic, and died (Fig. 1A). After exteriorization at E18.5, in vivo plethysmography never detected breathing pressure changes in Tshz $3^{\text {lac } Z \text { /lacZ }}$ embryos $(n=8)$ (Fig. $1 B$ ), even in two embryos in which one or two tiny respiratory efforts were occasionally seen at the time of exteriorization, whereas breathing movements were systematically recorded in WT littermates $\left(56 \pm 7 \mathrm{c} \cdot \min ^{-1} ; n=12\right.$ ) (Fig. $1 B$ ). To first exclude heart failure as the primary cause for neonatal death of $T s h z 3^{\text {laczllac }}$ animals, we recorded electrocardiograms of embryos in utero at E18.5. Heart rate was not significantly different in Tshz3 $3^{\text {lacZ/lacZ }}\left(66 \pm 13 \mathrm{c} \cdot \min ^{-1} ; n=4\right)$ and WT $(80 \pm 14$ $\left.c \cdot \min ^{-1} ; n=8\right)$. Postmortem lungs examination never revealed dilated lung alveoli in $T s h z 3^{\text {lacZ/lac } Z}$ mice (Fig. 1, compare $C, D$ ) despite a normal pattern of diaphragmatic innervation and neuromuscular junction formation (Fig. $1 F-K$ ). Furthermore, 
electromyographic discharges of chest respiratory muscles were recorded in WT but not in Tshz $3^{\text {lacZ/lacZ }}$ embryos (Fig. $1 E$ ). Therefore, given the absence of anomalies in the peripheral respiratory and cardiac systems, we suspected that $T s h z 3^{\text {lac } Z / l a c Z}$ have a defect in the central control for respiration.

We next compared the respiratory activity produced by the central respiratory network isolated in en bloc preparations at E18.5. Despite the absence of respiratory movements in Tshz3 mutant mice, bursts of activity could be recorded from phrenic and hypoglossal rootlets (Fig. $1 L)$. However, this activity was produced at a reduced frequency in $T s h z 3^{\text {lac } Z / l a c Z}$ ( $4.7 \pm 2.2$ burst $/ \mathrm{min} ; n=7)$ compared with WT $(10.6 \pm 1.4 \mathrm{burst} / \mathrm{min} ; n=10)$ and the duration of respiratory cycle was more variable in Tshz $3^{\text {lac } Z \text { /lacZ }}$ than WT ( $\sim 2.5$-fold increase). Interestingly, when the rib cage was transiently retained in vitro during the dissection, rhythmic movements of the rib cage were occasionally seen and rhythmic electromyographic discharges from chest muscles could be recorded in WT and Tshz $3^{\text {lacZ/lacZ }} \mathrm{em-}$ bryos (data not shown).

In en bloc preparations obtained from $T s h z 3^{\text {lacZ/lac }}$ embryos, the persistence of rhythmic phrenic bursts, chest movements, and chest muscles discharges in vitro versus their lack in vivo revealed that the mutant RRG was able to function when oxygenated in vitro, that the mutant chest muscles were innervated, and that their contractile properties were not affected by the $T \operatorname{sh} z 3$ mutation. The slow and variable rhythm of phrenic bursts in $T s h z 3^{\text {lacZ/lac } Z}$ in vitro preparations indicated that the inability of Tshz3 mutants to breath at birth originated, at least in part, from an alteration of the rhythmogenic properties of the RRG. However, the inability of $T \operatorname{sh} z 3$ mutants to breath at birth, as attested by in vivo flat plethysmographic traces and noninflated lungs, suggested that potentially other components of the central respiratory network were affected. These observations prompted us to characterize the expression of Tshz3 in motor nuclei during embryogenesis and in the RRG at developmental stages when the e-pF and preBötC are functional.

\section{TSHZ3 is expressed in a subset of developing}

\section{cranial motoneurons}

We analyzed the expression pattern of $T s h z 3$ in the hindbrain during embryonic development by performing in situ hybridization and X-Gal staining. As previously described, the temporal and spatial distribution of $\beta$-gal activity in $T s h z 3^{+/ l a c Z}$ is similar to the TSHZ3 protein (Caubit et al., 2008). This analysis revealed
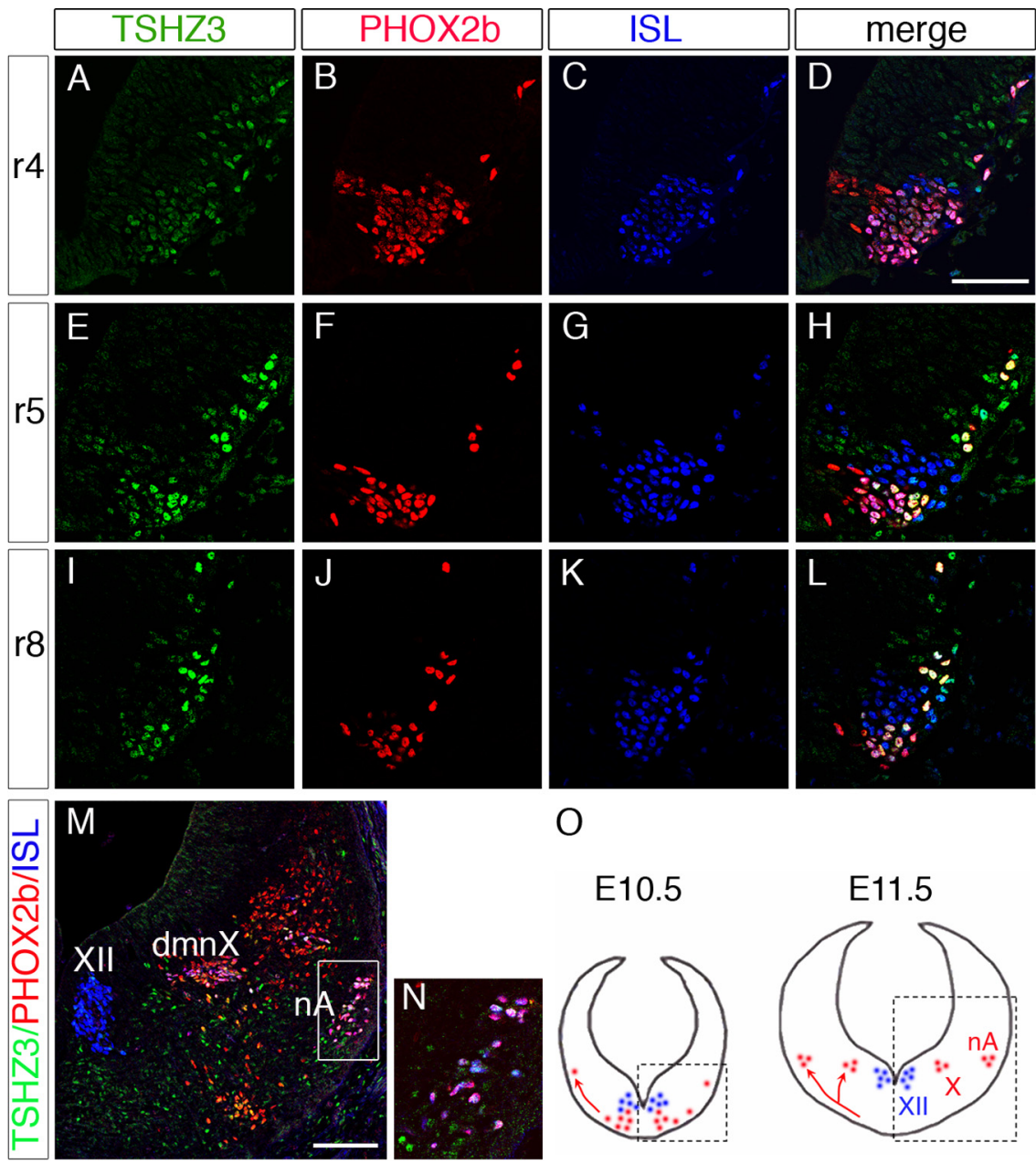

$\mathrm{O}$

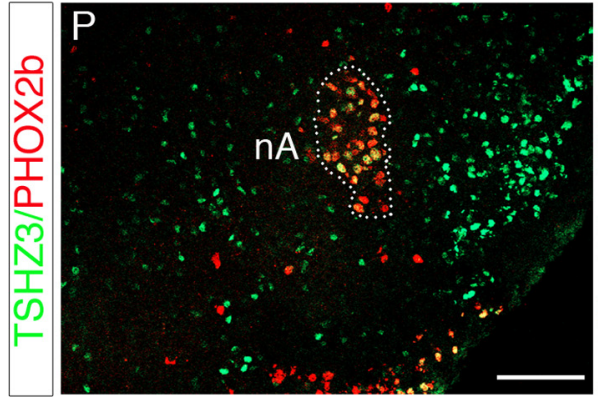

E10.5
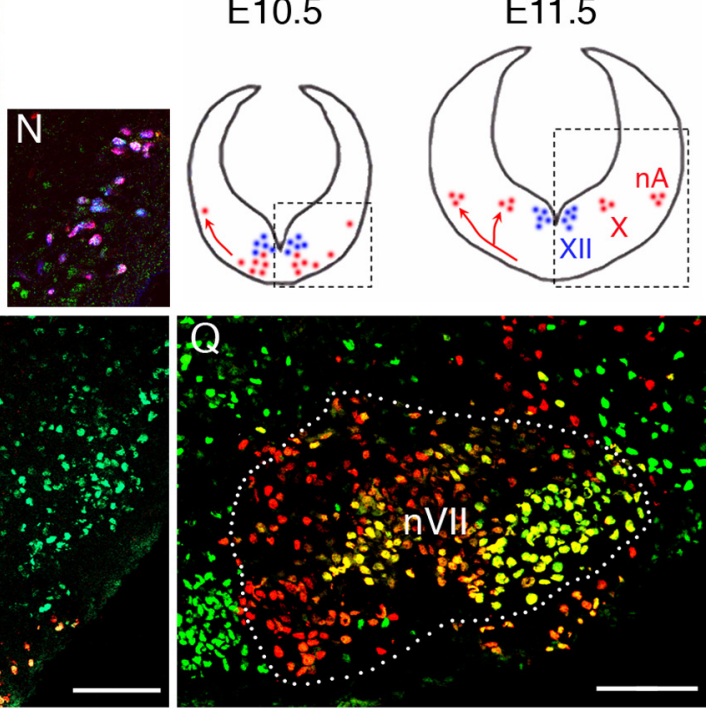

Figure 2. Comparative expression of TSHZ3 (green), PH0X2b (red), and ISLET1/2 (ISL) (blue) in rhombomeres (r) at stage E10.5. Transverse sections at the level of $\mathrm{r} 4(\boldsymbol{A}-\boldsymbol{D}), \mathrm{r} 5(\boldsymbol{E}-\boldsymbol{H})$, and $\mathrm{r} 8(\boldsymbol{I}-\boldsymbol{L})$. In $\mathrm{r} 5$ and $\mathrm{r} 8$, TSHZ3 is coexpressed with PH0X2b in a subset of ISL cells. $\boldsymbol{M}$, Immunodetection of TSHZ3, PHOX2b, and ISL at stage E11.5 showing expression of TSHZ3 in motorneurons of dmnX and nA. TSHZ3 is not detected in hypoglossal nucleus (XII). $\mathbf{N}$, Inset showing coexpression of TSHZ3, PHOX2b, and ISL in the precursors of $\mathrm{nA}$ boxed in $\boldsymbol{M}$. $\mathbf{0}$, Schematic representation of the origin and migratory behavior of the precursor of $\mathrm{dmnX}$ and $\mathrm{nA}$ (red) and XII (blue). The boxed areas are those photographed in $\boldsymbol{E}-\mathbf{M} . \mathbf{P}, \mathbf{Q}$, At E15.5, TSHZ3 is coexpressed with PHOX2b in motoneurons mainly located in the $\mathrm{nA}$ and in the lateral part of the facial nucleus (nVII) (yellow cells in $\boldsymbol{P}$ and $\mathbf{Q}$, respectively). $\mathrm{nVII}$ was delineated ( $\mathbf{Q}$, dotted line) based on ISL coimmunostaining (data not shown). Scale bars: $\boldsymbol{A}-\mathbf{L}, 50 \mu \mathrm{m} ; \boldsymbol{M}, \boldsymbol{P}, \boldsymbol{Q}, 100 \mu \mathrm{m}$.

(t)

that $T s h z 3$ was expressed early in the developing hindbrain, with an anterior limit of expression corresponding to the rhombomere (r) 3/4 boundary and in two medial columns of cells where cranial motoneurons are generated (supplemental Fig. S1, available at www.jneurosci.org as supplemental material). We compared at E10.5 the expression of TSHZ3 with ISLET1/2 (ISL) (a marker for motoneurons) and PHOX2b, which is expressed in branchio (bm)- and viscero (vm)-motoneurons (Pattyn et al., 1997) (Fig. 2). We found that TSHZ3 was present with PHOX2b in r4 (Fig. $2 A-D$ ), as well as at more caudal positions. For in- 
A
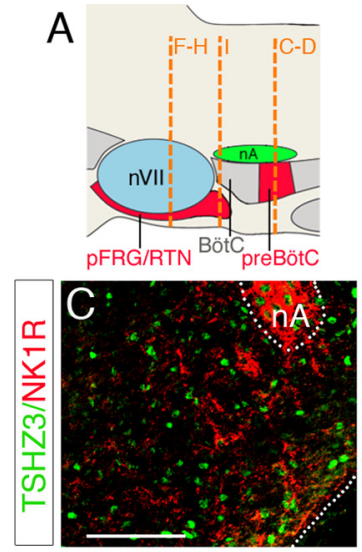

E
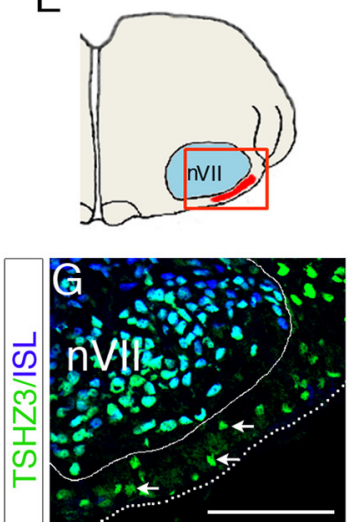
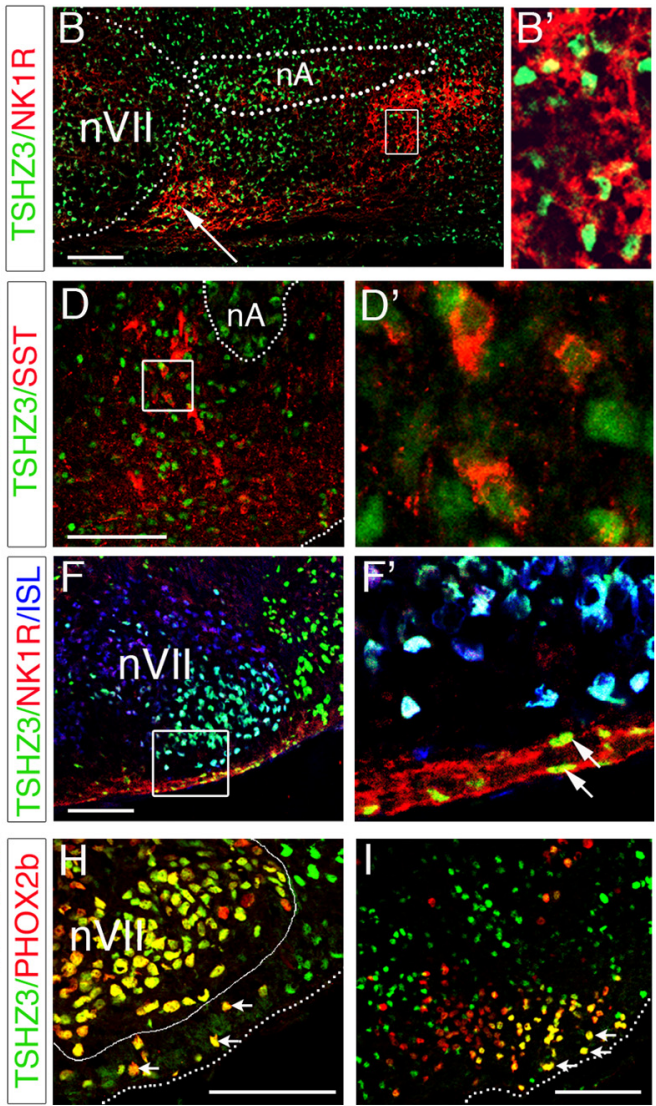

Figure 3. Expression of TSHZ3 in embryonic parafacial region and in preBötC. $A$, Schematic parasagittal view of the medullary ventral respiratory column indicating the position of the pFRG/RTN and the preBötC relative to facial nucleus (nVII) and $n A$ respectively. BötC, Bötzinger complex. The dashed lines show the position of the transverse sections through the $\mathrm{nVII}(\boldsymbol{F}-\boldsymbol{H})$, immediately caudal to $\mathrm{nVII}(\boldsymbol{I})$ and in the preBötC $(\boldsymbol{B}-\boldsymbol{D})$. $\boldsymbol{B}$, Parasagittal section crossing the lateral part of the $\mathrm{nVII}$ at E15.5. TSHZ3 is expressed in the nVII as well as in NK1R-positive cells located caudally to the nVII (white arrow), and the dotted lines indicate the position of the nVII and the nA. TSHZ3 + cells are also found in the preBötC. The inset shows that TSHZ3 + cells expressed NK1R. C, Transverse section of an E15.5 hindbrain showing expression of TSHZ3 in the NK1R-positive neurons of the preBötC. D, Transverse section of E18.5 hindbrain showing expression of TSHZ3 and SST; the dotted line delineates the nA. $\boldsymbol{D}^{\prime}$, Magnification of TSHZ3-positive cells from the box in $\boldsymbol{D}$. $\boldsymbol{E}$, Schematic transversal view indicating the position of sections in $\boldsymbol{F}$ - $\boldsymbol{H}$. $\boldsymbol{F}$, Coexpression of TSHZ3 and NK1R in cells located ventrally to the nVII, a structure immunopositive for ISL (blue). $\boldsymbol{F}^{\prime}$, Magnification of box in $\boldsymbol{F}$. G, $\boldsymbol{H}$, Section through the E15.5 hindbrain, used for detection of TSHZ3 and ISL $(\boldsymbol{G})$ and TSHZ3 and PHOX2b $(\boldsymbol{H})$. The white arrows indicate TSHZ3 + cells expressing PHOX2b in $\boldsymbol{H}$; these same cells do not express ISL ( $\boldsymbol{G}$, white arrows). A white line delineates nVII, and a dotted line marks the ventral medullary surface. $I$, Transverse section performed immediately caudal to the nVII at E15.5, showing expression of TSHZ3 in the most laterally located PHOX2b + cells (arrows). Scale bars, $100 \mu \mathrm{m}$.

stance, in $\mathrm{r} 5$ (Fig. $2 E-H$ ) and in the caudal most part of the hindbrain (r8) (Fig. 2I-L), TSHZ3 marked a subset of ISLpositive cells that occupy the most ventral position. TSHZ3 was never detected in the neuroepithelium and TSHZ3 + cells were always located in the lateral aspects of the neural tube (close to the pial surface). Thus, at all axial levels, TSHZ3 was coexpressed with $\mathrm{PHOX} 2 \mathrm{~b}$ in postmitotic bm and vm motoneurons. Coimmunodetection of TSHZ3 with Nkx2.2 (data not shown) confirmed that TSHZ3 + cells located ventrally in the mantle layer derived from the vm/bm neuron progenitor (p3) domain (Guthrie, 2007). At E11.5, the ventral bm/vm neurons in the caudal hindbrain had migrated dorsally to form the dorsal motor nucleus of the vagus nerve (dmnX) and the nA. At this stage, the cells coexpress PHOX2b, ISL (Pattyn et al., 1997; Dauger et al., 2003), and TSHZ3 (Fig. $2 M, N$ ). In contrast, the ISL+/PHOX2b- somatic motoneurons of the hypoglossal nucleus did not express TSHZ3 (Fig. 2M). By E15.5, TSHZ3 was no longer expressed in the dmnX nucleus (data not shown), whereas it persisted in the $\mathrm{nA}$ (Fig. 2P). TSHZ3 expression was also detected in a subset of mo-

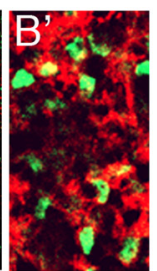

toneurons located in the central and the lateral most part of the nVII (Fig. 2Q). The expression pattern of TSHZ3 is therefore consistent with its playing a role in the specification and differentiation of bm/vm neurons involved in airway control.

\section{TSHZ3 is expressed in the e-pF oscillator and in the pre-Bötzinger complex}

In an attempt to explain the slower respiratory rhythm generated by the $T s h z 3^{\text {lac } Z \text { /lac } Z}$ preparations, we also examined the pattern of expression of $T s h z 3$ in the two main neuronal groups directly involved in the respiratory rhythmogenesis (i.e., the e-pF and the preBötC) (Fig. 3A). The e-pF lays ventrally to the nVII and is immunoreactive for NK1R, PHOX2b, and Vglut2 (Dubreuil et al., 2008; Thoby-Brisson et al., 2009). The preBötC, located ventrally to the $\mathrm{nA}$, is known to be immunoreactive for NK1R (Gray et al., 1999) and somatostatin (Stornetta et al., 2003). Immunostainings performed on parasagittal and transverse sections at E15.5 and E18.5 showed that TSHZ3 + cells were located in the NK1R+ region (Fig. $3 B, B^{\prime}, C$ ), and some of them coexpressed somatostatin (Fig. $3 D, D^{\prime}$ ). TSHZ3 was also detected in a cluster of NK1R+ cells extending caudally from the posterior limit of the nVII (Fig. 3B, arrow). Frontal sections performed at E15.5 crossing the nVII (Fig. $3 E$ ) showed that TSHZ3 is also coexpressed with NK1R in cells located close to the medullary surface under the lateral part of the nVII (Fig. $3 F ; F^{\prime}$, arrows). At this stage TSHZ3 + cells were also identified as PHOX2b +/ISL - cells, as expected for e-pF neurons (Fig. 3G, $H$, arrows). Under the lateral part of the nVII, most of PHOX2b + cells expressed TSHZ3 (92\%; 20 sections from two embryos). Caudally to nVII, TSHZ3 was expressed in a lateral subset of PHOX2b-expressing cells (Fig. 3I, arrows). TSHZ3 was also detected in other nuclei involved in the modulation of respiratory rhythm, such as the nucleus of the solitary tract and the Raphe pallidus (supplemental Fig. S1G, available at www.jneurosci.org as supplemental material). Together, these observations indicated that, during development, TSHZ3 was expressed, but not exclusively, in neurons constituting the two main respiratory oscillators. We then performed experiments to further characterize the mechanisms responsible for the abnormal respiratory rhythm and to identify the additional cause of the neonatal inability to breathe. In the light of the expression pattern reported above, we focused our attention on the $\mathrm{nA}$ and on the RRG.

\section{Tshz3 mutation impairs nucleus ambiguus development} We first analyzed the anatomical consequences of Tshz3 inactivation on the development of the hindbrain motor nuclei. Study of early Phox $2 b$ and neurofilament expression suggested that, in 
the absence of $T s h z 3$, the specification of hindbrain rhombomeres was unaffected (supplemental Fig. S2, available at www. jneurosci.org as supplemental material). However, in E18.5 homozygous mutants, although the trigeminal nucleus, nVII, $\mathrm{dmnX}$, and the hypoglossal nuclei could be detected using Nissl staining and gross hindbrain structure was unaffected (data not shown), the nA was not detectable (Fig. $4 A, B$ ). This was confirmed using in situ hybridization for choline acetyltransferase (data not shown) or peripherin. No significant differences in the size and the organization of the nVII and the other upper airway motor nuclei could be detected between WT and mutant embryos (Fig. $4 D, E, G, H)$. However, a drastic reduction in size of the $\mathrm{nA}$ was apparent at E18.5 (Fig. 4C,F). To understand the origin of the reduced number of $\mathrm{nA}$ neurons at E18.5, we examined earlier developmental stages in the mutants. Already by E15.5, the number of PHOX2b-expressing cells in the mutant nA was drastically reduced compared with WT (Fig. 4I,J). This reduction is $\sim 75 \%$, as revealed by quantitative analysis $(758 \pm 26$ and $167 \pm 47$ PHOX2B-positive cells per $\mathrm{nA}$ for six WT and six Tshz $3^{\text {lacZ/lacZ }}$ E15.5 embryos, respectively; $p=0.000036$ ). To determine whether cell death contributed to the reduction in motoneuron numbers, we performed terminal deoxynucleotidyl transferase-mediated biotinylated dUTP nick end labeling (TUNEL) at E14.5. The number of apoptotic cells specifically located in the nA was significantly higher in Tshz3 mutants compared with WT. This was quantified by performing double immunostaining for $\mathrm{PHOX} 2 \mathrm{~b}$ and activated caspase-3 at E14.5 (Fig. $4 M, N$ ); there were $\sim 2.5$-fold more degenerating motoneurons in $\mathrm{nA}$ in $T$ sh $z 3$ mutants compared with WT $(23 \pm 6$ and $62 \pm 14$ activated caspase-3-positive cells per nA for four WT and four Tshz $3^{\text {lacZ/lacZ }}$ E14.5 embryos, respectively; $p=0.0023$ ). In contrast, no increase in cell death could be detected in the nVII of Tshz3 mutants. Together, these data show that $T s h z 3$ mutation specifically affects the survival of the motoneurons forming the $\mathrm{nA}$. The drastic reduction in the number of motoneurons innervating the upper airway apparatus would be expected to lead to highly resistive breathing and could potentially explain why, despite the generation of some respiratory rhythms, lung alveoli were never found to be inflated in $T$ shz $3^{\text {lac } Z / l a c Z}$ neonates.

\section{Functional and anatomical analysis of the two respiratory} oscillators at the time of their emergence

The abnormal respiratory rhythm observed in Tshz3 mutant embryos in vitro and the patterns of expression of TSHZ3 suggested a potential role for $T s h z 3$ in the functional establishment of the RRG during fetal development. We therefore examined the functional status of the e-pF and the preBötC in Tshz3 mutants by re- cording spontaneous rhythmic activity from both oscillators at the time of their functional emergence, at E14.5 for the e-pF and E15.5 for the preBötC, respectively (Thoby-Brisson et al., 2005, 2009). Spontaneous rhythmic activities were analyzed using calcium imaging performed on isolated en bloc brainstem preparations for the $\mathrm{e}-\mathrm{pF}$ and brainstem transverse slice preparations for the preBötC.

\section{Dysfunction of the e-pF oscillator in the Tshz3 mutant}

Calcium imaging performed at E14.5 on isolated hindbrain preparations obtained from WT embryos revealed spontaneous rhythmic fluorescent changes occurring in the e-pF region (Fig. $5 A$ ), which correspond to the synchronized activity of the e-pF neurons (Thoby-Brisson et al., 2009). As shown in previous studies (Dubreuil et al., 2009; Thoby-Brisson et al., 2009), lowering the $\mathrm{pH}$ of the bathing solution triggered a marked increase in the e-pF frequency activity of WT embryos from $10.7 \pm 0.6$ burst/ $\min$ in $\mathrm{pH} 7.4$ to $17.7 \pm 0.8 \mathrm{burst} / \mathrm{min}\left(n=14 ; p=10^{-7}\right)$ in $\mathrm{pH}$ 7.2 (Fig. $5 A, C$ ). In contrast, in $T s h z 3^{\text {lac } Z / l a c Z}$ embryos at the same age, no rhythmically organized activity could be detected in 

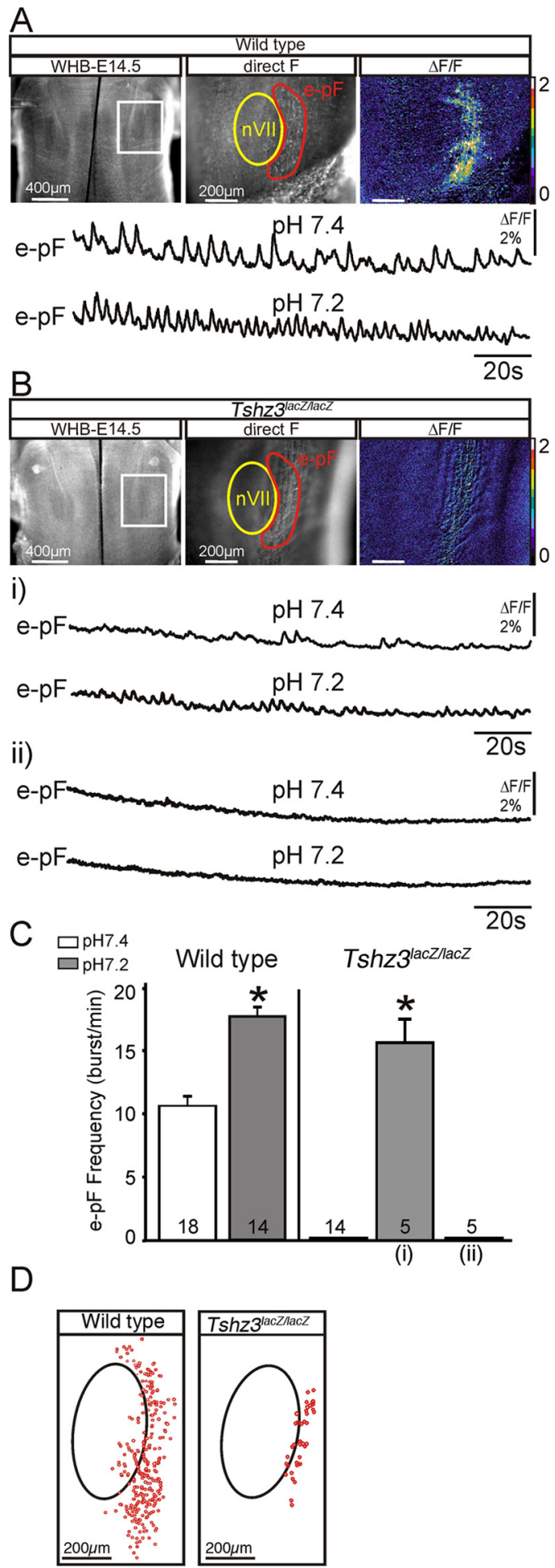

Figure 5. Dysfunction of the e-pF oscillator at E14.5 in Tshzz ${ }^{\text {lac } / l a c z}$ embryos. A, Photomicrographs of a whole hindbrain preparation (WHB) (ventral view) obtained from an E14.5 WT embryo loaded with Calcium Green-1 AM observed in direct fluorescence at low (left panel) and higher magnification (middle panel). The white rectangle delimits the e-pF area observed at a higher magnification in the two right panels. The facial motor nucleus (nVII) and the e-pF region are outlined in yellow and red, respectively. The rightmost panel illustrates spontaneous calcium transients occurring over the parafacial area as relative changes in fluorescence $(\Delta F / F)$. The traces below correspond to transient fluorescence changes recorded in the e-pF region in control conditions, pH 7.4 (top trace), and in pH 7.2 (bottom trace). $\boldsymbol{B}$. Same legend as in $\boldsymbol{A}$ for

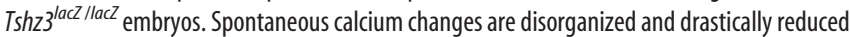
in some preparations (i, top trace) or completely absent in others (ii, top trace). The bottom traces in $i$ and $i i$ show the response to acidification. Only preparations showing sporadic calcium control conditions in the e-pF region. Although one-half of the preparations ( 9 of 18) exhibited some sporadic, weak, and unorganized fluorescent changes (Fig. 5Bi), the other one-half showed no activity at all (Fig. 5Bii). Five preparations of each group were exposed to $\mathrm{pH}$ challenges. In the first group, lowering the $\mathrm{pH}$ to 7.2 triggered some weak fluorescent transients that were partially rhythmically organized with a frequency of $15.4 \pm$ 1.4 burst/min (Fig. 5Bi,C). In the second group, the $\mathrm{pH}$ challenge failed to initiate activity and had no apparent effect (Fig. 5Bii,C). A closer examination of the e-pF region was then performed to detect individual rhythmically active neurons. A representative map of the active cells is shown in Figure $5 D$ for a WT and a $T s h z 3^{\text {lac } Z \text { /lac } Z}$ preparation. The number of rhythmically active cells in the Tsh $z 3$ mutants ( $24 \pm 3$ cells/e-pF; $n=6$ preparations) was strongly reduced compared with the WT $(213 \pm 32$ cells/ e-pF; $n=5$ preparations; $p=10^{-4}$ ) (Fig. $5 D$ ). The very low number of active e-pF cells in Tshz $3^{\text {lacZ/lacZ }}$ embryos generally precluded the detection of rhythmic population calcium activity. Note that these numbers of active cells represent only cells that have taken up the dye, that are rhythmically active, and that can be detected in brainstem preparation. Together, these data show that the e-pF oscillator is severely functionally impaired in the Tshz3 mutants.

The e-pF oscillator is anatomically preserved in the Tshz3 mutant The reduced number of active cells in the e-pF of Tshz $3^{\text {lac Z lac } Z}$ preparations prompted a search for anatomical defects. We analyzed the expression of several e-pF markers such as PHOX2b, NK1R, LBX1, vGlut2, and Atoh1 (Pagliardini et al., 2008; Dubreuil et al., 2009; Rose et al., 2009; Thoby-Brisson et al., 2009) in the e-pF region of mutant embryos at E15.5. PHOX2b+/ $\mathrm{NK} 1 \mathrm{R}+$ cells were present in the e-pF region (Fig. $6 A-D)$. The number of PHOX2b/NK1R double-positive cells was estimated per one parafacial region for six WT and six Tshz $3^{\text {lacZ/lacZ }}$ E15.5 embryos. Cells were counted into two groups, one ventral to the $\mathrm{nVII}$ and the other forming a compact group extending caudally to $\mathrm{nVII}$. The quantification revealed no significant differences between both genotypes ventrally $(234 \pm 54$ vs $221 \pm 45$; $p=$ $0.87)$ and caudally (746 \pm 154 vs $702 \pm 130 ; p=0.46)$. Similar observations were made for LBX1+ neurons in Tshz3 mutants (data not shown) and for Atoh1, expressed in cells close to the medullary surface located ventrolaterally to the nVII (Fig. 6E,F) and extending more caudally (Fig. 6G,H). Finally, vGlut2expressing cells located ventrally and caudally to the nVII were clearly identified in Tshz3 mutants (Fig. 6I,J). The presence and normal pattern of all e-pF markers tested argue for the anatomical preservation of this respiratory oscillator.

Alteration of bursting properties of e-pF neurons in the Tshz3 mutant

Despite the anatomical presence of the e-pF cells, rhythmic activity could not be detected in the e-pF oscillator, suggesting that

$\leftarrow$

changes in pH 7.4 exhibit more or less rhythmically organized weak calcium changes in pH 7.2.

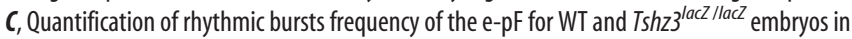
control conditions at pH 7.4 (white bars) and at pH 7.2 (gray bars). For the mutants, the response to acidification has been divided in two columns to distinguish between completely silent preparations [rightmost column (ii)] and the slightly more active ones [middle column (i)]. The number of preparations analyzed in different conditions is indicated on each bar. $\boldsymbol{D}$, Maps of rhythmically active cells (red circles) for a WT preparation (left panel) and a $T s h z 3^{\text {lacz /lacz }}$ preparation (middle panel). The black ovals indicate the position of the $n V I I$. The graph (right) shows the quantification of the mean number of rhythmically active cells detected in five WT (white bars) and six Tshz3 mutant (black bars) preparations. The asterisks indicate significantly different values: $p<0.05$. Values are given as mean \pm SEM. 


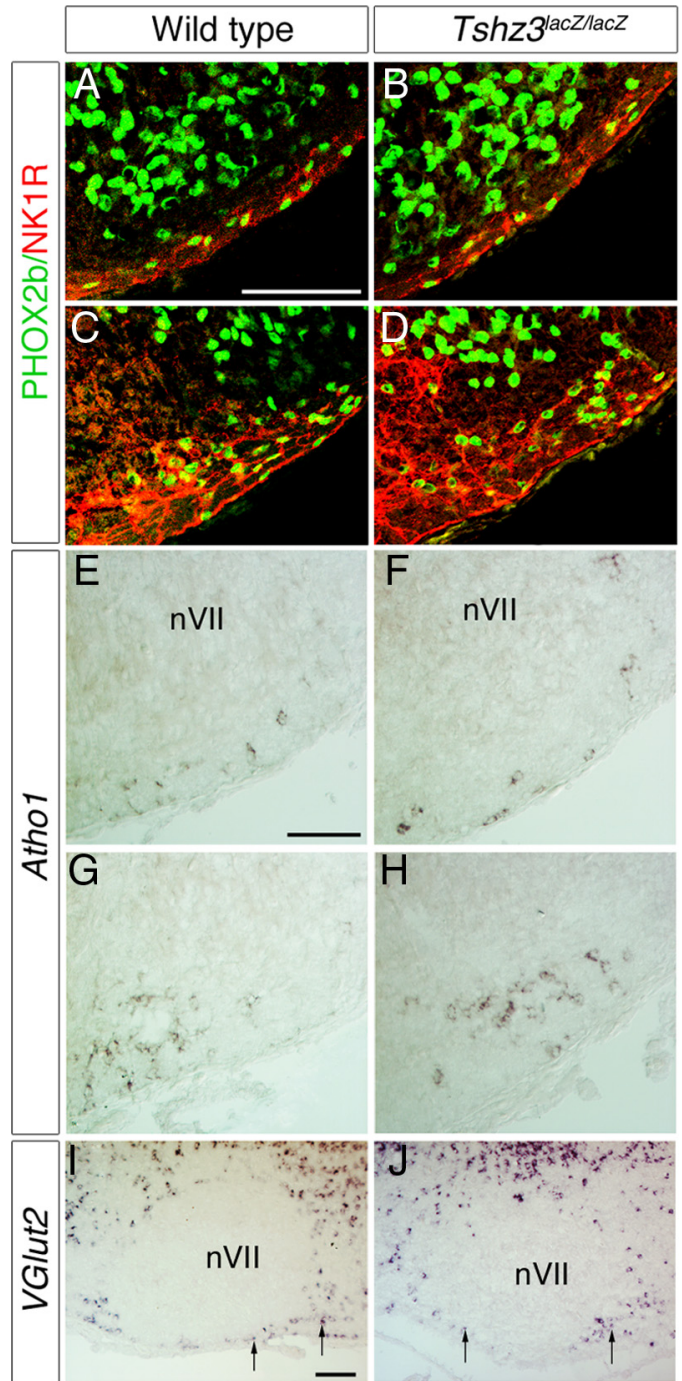

Figure 6. Anatomical analysis of the e-pF in WT and $T s h z 3$ mutants. A-D, Immunodetection of PHOX2b (green), NK1R (red), and ISL (purple) on transverse section from E15.5 WT $(A, C$ and mutant embryos $(\boldsymbol{B}, \boldsymbol{D})$. Analysis of these markers is shown at two axial levels through the facial nucleus, at mid part $(\boldsymbol{A}, \boldsymbol{B})$, and at its caudal extremity $(\boldsymbol{C}, \boldsymbol{D}) . \boldsymbol{E}-\boldsymbol{H}$, In situ hybridization showing expression of Atoh1 on transverse sections through the hindbrain of a WT $(\boldsymbol{E}, \boldsymbol{F})$ and a $T$ shz3 mutant $(\boldsymbol{F}, \boldsymbol{H})$ at E15.5. Atoh 1 expression persists in mutants. $\boldsymbol{I}, \boldsymbol{J}$, In situ hybridization showing expression of $v G l u t 2$ on parasagittal sections through hindbrain of WT (I) and Tshz3 mutant $(\boldsymbol{I})$ at P0. vGlut2 + cells were found at a similar location, close to the medullary surface, ventral and caudal (arrows) to the nVII. Scale bars, $100 \mu \mathrm{m}$.

intrinsic bursting properties of individual e-pF neurons might be altered in the Tshz3 mutants. To test this, we investigated the cellular properties of the e-pF neurons in WT animals and in $T s h z 3$ mutants using the whole-cell patch-clamp recording approach. In E14.5 WT hindbrain preparations, e-pF neurons $(n=$ 18) could first be identified by their ability to generate rhythmic bursts of action potentials in phase with the e-pF population activity and in a strong voltage-dependent manner (Fig. 7A). At resting membrane potential, individual bursts of activity in e-pF cells are highly reproducible all-or-none events (Fig. 7A, left box) (Thoby-Brisson et al., 2009). The e-pF identity of 11 recorded neurons was subsequently confirmed by biocytin cellular fills and PHOX2b counterstaining (Fig. 7A) (Thoby-Brisson et al., 2009). In contrast, in $T s h z 3$ mutant preparations, no rhythmic activity could be detected in the e-pF population, denoting a functionally deficient e-pF oscillator (Fig. $7 B$ ). In addition, individual e-pF neurons exhibited abnormal bursting activity. Of 18 recorded neurons, 4 failed to show a bursting pattern of discharge, generating instead only individual spikes; these were excluded from additional study. The remaining 14 neurons featured an abnormal, but still voltage-dependent, bursting activity composed of events with variable duration (Fig. 7B). Many bursts showed premature termination because of the failure to maintain a depolarized potential, thus resulting in a shortening of the burst duration. The average burst duration was nearly threefold shorter in mutant preparations: $683 \pm 34 \mathrm{~ms}$ in WT $(n=60$ events, 7

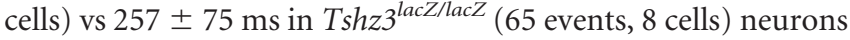
$\left(p<10^{-7}\right)$. These data suggested that as-yet-unidentified intrinsic membrane properties were affected in Tshz $3^{\text {lac } Z \text { /lac } Z}$ embryos. However, no significant differences in the resting potential $(-46.4 \pm 0.9 \mathrm{mV}, n=18$ in WT; and $-45.8 \pm 0.8 \mathrm{mV}, n=14 \mathrm{in}$ Tshz $3^{\text {lac Z lac }}$ embryos $)$ or the membrane resistance $(2105 \pm 158$ $\mathrm{M} \Omega, n=18$ in WT; and $2234 \pm 237 \mathrm{M} \Omega, n=14$ in Tshz $3^{\text {lacz/lac }}$ embryos) could be found between WT and Tshz $3^{\text {lacZ/lac } Z}$ neurons. Some neurons loaded with biocytin during the patch-clamp recording session were later processed for immunostaining against PHOX2b and $\beta$-gal. All (10 of 10) neurons tested were triple-positive (Fig. $7 B$ ) and thus complied with the chemical code characterizing e-pF neurons (Dubreuil et al., 2009). Together, these data showed that the functional deficiency of the e-pF oscillator is associated with altered bursting properties of its constitutive neurons.

\section{The preBötC is not affected by the Tshz3 mutation}

The fact that TSHZ3 is expressed in the preBötC region prompted us to also test the functional and anatomical status of this respiratory oscillator in the $T s h z 3^{\text {lac } Z / l a c Z}$ embryos. Using calcium imaging and electrophysiological recordings (data not shown) on transverse brainstem slices obtained at E15.5 from Tshz $3^{\text {lacZ/lac Z }}$ and WT littermates, we sought to detect rhythmically organized neuronal activity. Our recordings revealed the presence of spontaneous rhythmic activity in the regions of the bilaterally distributed preBötC in slices obtained from both genotypes (Fig. 8A,B). Moreover, bursting frequencies were comparable between WT and Tshz $3^{\text {lacZ/lacz }}$ embryos $(8.5 \pm 2.2 \mathrm{burst} / \mathrm{min}$ in WT, $n=10$; and $8.1 \pm 2.7 \mathrm{burst} / \mathrm{min}$ in the Tshz $3^{\text {lac } Z / l a c Z}$ embryos, $n=8 ; p=$ $0.9)$. No significant differences in the activities of the preBötC across genotypes could be noted at later developmental stages (E16.5 and E18.5) (data not shown) using electrophysiological recordings. Moreover, anatomical markers of preBötC oscillator cells, such as NK1R and somatostatin, were unaffected by the Tshz3 mutation (Fig. $8 C-F^{\prime}$ ). So, in contrast to the e-pF, the preBötC emerged and developed normally in Tshz $3^{\text {lac } Z / l a c Z}$ embryos.

\section{Defects in respiratory rhythm and $\mathrm{pH}$ sensitivity in $T \operatorname{sh} z 3$ mutant embryos}

At approximately E15.5, the e-pF and preBötC oscillators are known to couple to form the RRG and generate the appropriate respiratory-like behavior (Thoby-Brisson et al., 2009). We therefore assessed the activity of the RRG by recording from phrenic nerve roots (C4) in isolated brainstem-spinal cord preparations obtained from WT and Tshz3 $3^{\text {lacZ/lacZ }}$ littermates at E16.5 (Fig. $9 A)$. In WT preparations, $\mathrm{C} 4$ recordings indicated the presence of a spontaneously active RRG at a frequency of $8.8 \pm 0.9 \mathrm{burst} / \mathrm{min}$ $(n=9)$ (Fig. 9A,C). In mutant preparations, the RRG had a reduced frequency $(4.8 \pm 0.6$ burst $/ \mathrm{min} ; n=9$ ) (Fig. 9B, $C$ ). This abnormally slow respiratory-like rhythm was maintained at later developmental stages. At E18.5, the mean frequency was $10.6 \pm$ $1.4 \mathrm{burst} / \mathrm{min}$ in the WT $(n=10)$ and $4.7 \pm 2.2 \mathrm{burst} / \mathrm{min}$ in the 


\section{A Wild type}

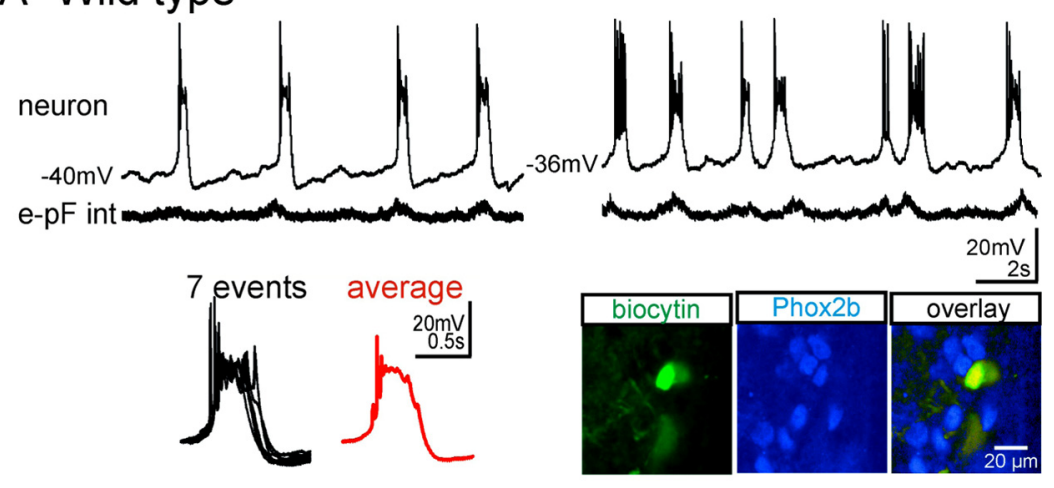

\section{B Tshz3laczllacz}

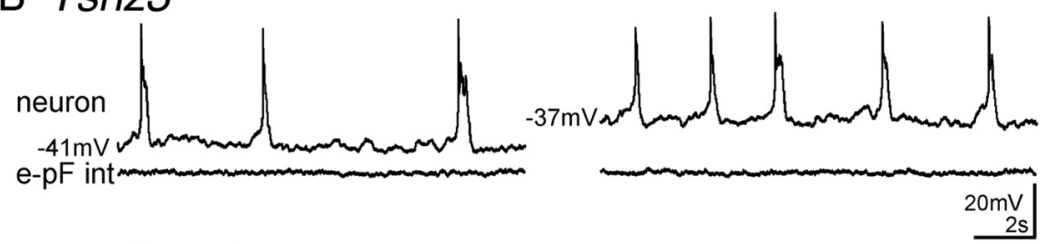

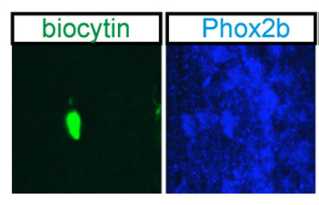

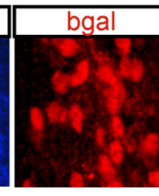

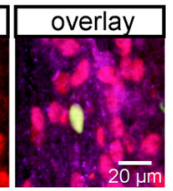

Figure 7. The bursting properties of the e-pF neurons are altered at E14.5 in the Tshz3 mutant. A, Membrane trajectory (top traces) of an e-pF neuron recorded at two different resting potentials (left and right traces) recorded simultaneously with the e-pF population integrated activity (bottom traces). Seven superimposed bursts and the corresponding average trace (red) are illustrated at an extended time scale in the black box. The three bottom right panels represent the immunolabeling for PHOX2b (blue) for a biocytin-filled (green) e-pF neuron previously electrophysiologically identified with a patch-clamp recording. $\boldsymbol{B}$, Same legend as in $\boldsymbol{A}$ for an e-pF neuron recorded from a Tshz3 mutant preparation. The neuron generates abnormal bursts, and the duration and the shape of the bursts are variable from one event to the other (see the box). Immunolabeling for PHOX2b (blue) and $\beta$-gal (red) ascertained the identity of the recorded neuron filled with biocytin (green).

Tshz $3^{\text {laczlacz }}$ embryos $(n=7)$ (Fig. 9C). This slow respiratory rhythm was reminiscent of that found in other mutants that show selective deficiency of the e-pF oscillator, thus lacking the e-pF entrainment of the preBötC normally tuning up the frequency of the RRG (Dubreuil et al., 2009; Thoby-Brisson et al., 2009).

Another role of the e-pF in $\mathrm{CO}_{2}$ sensing and/or in providing chemosensory drive to the RRG has been proposed, so we next investigated whether $\mathrm{pH}$ sensitivity was normal in Tshz $3^{\text {lacZ/lac } Z}$ embryos at E16.5, a stage at which both e-pF and preBötC oscillators were functional, and at E18.5, the latest embryonic stage preceding birth. En bloc medullary preparations were subjected to mild acidification by reducing the $\mathrm{pH}$ from 7.4 to 7.2 (Fig. $9 A, C)$. At E16.5, acidification significantly $\left(p=6 \times 10^{-4}\right)$ increased the phrenic burst frequency in WT preparations $(n=9)$ from $8.5 \pm 0.9 \mathrm{burst} / \mathrm{min}$ in $\mathrm{pH} 7.4$ to $14.4 \pm 1 \mathrm{burst} / \mathrm{min}$ in $\mathrm{pH}$ 7.2 but insignificantly $(p=0.13)$ increased the frequency in Tshz $3^{\text {lacZ/lacZ }}$ preparations $(n=9)$ from $5.3 \pm 0.7 \mathrm{burst} / \mathrm{min}$ in pH 7.4 to $7.8 \pm 1$ burst/min in pH 7.2 (Fig. $9 B, C$ ). At E18.5, acidification still significantly $(p=0.04)$ increased the phrenic burst frequency in $10 \mathrm{WT}$ preparations from $10.3 \pm 1.5$ burst/ $\mathrm{min}$ in $\mathrm{pH} 7.4$ to $13.6 \pm 1.4$ burst $/ \mathrm{min}$ in $\mathrm{pH} 7.2 \mathrm{but} \mathrm{had} \mathrm{no} \mathrm{effect}$ $(p=0.29)$ at all in seven Tshz $3^{\text {lacZ/lacZ }}$ preparations (4.7 \pm 2.3 burst $/ \mathrm{min}$ at $\mathrm{pH} 7.4$ and $5.2 \pm 2.9 \mathrm{burst} / \mathrm{min}$ at $\mathrm{pH}$ 7.2). Slow respiratory rhythm and loss of response to acidification were also observed in more physiological conditions (i.e., $5 \mathrm{~mm}$ external potassium) (data not shown). These data demonstrated the deficiency of the Tshz $3^{\text {laczllacz }} \mathrm{RRG}$ to respond to acidification. This

anomaly likely contributes to the inability of $T s h z 3^{\text {lac } Z \text { /lac } Z}$ neonates to produce the first breath at birth.

Because TSHZ3 is expressed in raphe neurons (supplemental Fig. S1, available at www.jneurosci.org as supplemental material) and because serotoninergic (5HT) neurons may contribute to central chemoception (Nattie et al., 2004; Richerson, 2004; Nattie, 2006), we examined whether Tshz3 mutation affects the 5-HT neuronal population. We estimated and compared the total number of 5-HTpositive cells in the medulla oblongata in E16.5 WT and Tshz3 $3^{\text {lacZ/lacZ }}$ embryos. The medullary 5-HT neurons were present in comparable numbers in the WT and the Tshz3 mutants in both the medullary raphe and the ventrolateral medulla (supplemental Fig. S2 $E-G$, available at www. jneurosci.org as supplemental material). Furthermore, NK1R immunostaining did not reveal anatomical defects in the medullary raphe (supplemental Fig. S2H, $I$, available at www.jneurosci.org as supplemental material). Consequently, the defect in chemosensitivity detected in $T s h z 3$ mutants could not be attributed to 5-HT cell loss but mainly results from perturbed rhythmogenic properties within the e-pF oscillator. Thus, Tsh $z 3$ mutant embryos displayed a slowed-down respiratory rhythm and a loss of chemosensitivity at E16.5 onward, which contributed to their inability to secure homeostasis after umbilical cord interruption at birth or at exteriorization.

\section{Discussion}

Functional breathing requires the coordinated development of multiple central and peripheral neuronal circuits. Our results indicate that loss of function of a single gene, $T s h z 3$, is sufficient both to prevent survival of a subset of cranial motoneurons (the $\mathrm{nA}$ ) and to disturb the functional emergence of one respiratory oscillator (the e-pF). The combination of these deficits at embryonic stages results in a severe dysfunction of the RRG and of the upper respiratory tract, which is not compatible with survival at birth. Thus, TSHZ3 plays a major role in coordinating the development of systems that generate respiratory rhythms with those involved in airway opening.

\section{Tshz3 mutation impairs survival of motoneurons of the nucleus ambiguus}

Compelling evidence exists that the motor act of breathing not only requires rhythmic contractions of chest pump muscles to induce airflow into and out of the lungs but also coordinated contractions of complex upper airway muscles to modulate airflow and more importantly prevent airway collapses during powerful diaphragmatic contractions, especially in neonates. The nA contains pharyngeal and laryngeal motoneurons that govern the upper airway valve as well as motoneurons innervating the esophagus, all of which are involved in respiratory and swallowing behaviors (Bieger and Hopkins, 1987; Standish et al., 1994). 
The survival requirements of $\mathrm{nA}$ motoneurons have not been widely studied. Inactivation of the genes for the cytokine receptor subunits LIFR (leukemia inhibitory factor receptor) (Li et al., 1995) or gp130 (Nakashima et al., 1999) leads to loss of nA motoneurons, whereas exogenous neurotrophic factors can protect nA motoneurons against the effects of axotomy (Araki et al., 2006). However, similar effects are observed for neighboring cranial motor nuclei such as nVII. Here, we show that TSHZ3 is required for the survival of a significant proportion of $\mathrm{nA}$ motoneurons in a manner that is not shared by other cranial nuclei. Our data show that the formation of bm/vm precursors and their migration toward their final position, leading to formation of structured motor nuclei, are not dependent on Tshz3 function. Thus, unlike $P h o \times 2 b$, which is required early for the formation of all bm and cranial vm neurons (Pattyn et al., 2000), Tshz3 is required for the maintenance of $n A$ motoneurons only once they have reached their final position. In Tshz3 mutant, the loss of nA motoneurons was caused by a significant increase of apoptosis. This increase happens in a period of naturally occurring developmental cell death (approximately E14.5) and critical for the formation of upper respiratory tract neuromuscular control. The mechanisms leading to the death of nA motoneurons in Tshz3 mutants are unknown; however, TSHZ3 may participate directly (in a cell-autonomous manner) in intracellular signaling important for survival and/or maturation of embryonic motoneurons. Interestingly, Kajiwara et al. (2009) showed that a reduced expression of TSHZ3 correlates with an increase of the primate-specific caspase- 4 expression, leading to the progression of Alzheimer's disease. TSHZ3 can participate to repressor complexes via a direct association with the promoter region of casp4, suggesting that TSHZ3 may protect neurons from apoptosis in a cell-autonomous way. It is not known whether in the mouse a TSHZ-caspase pathway exists and conditional inactivation of Tshz3 will help to elucidate the role of Tshz3 in neuroprotection. Strikingly, whereas TSHZ3 is detected in several motor nuclei, nA motoneurons are selectively vulnerable to the Tshz3 mutation. Comparative analysis of the expression of the three members of the Tshz gene family may help to determine whether survival of motor nuclei in Tshz3 mutant could rely on the expression of other $T s h z$ genes.

Overall, the massive loss of nA motoneurons induced by Tshz3 mutation probably impairs the neural control of upper airways, thus explaining the observed absence of pressure changes and the collapsed lung alveoli in the mutants. This functional deficit is partly responsible for the complete lack of ventilation observed in the mutant animals.
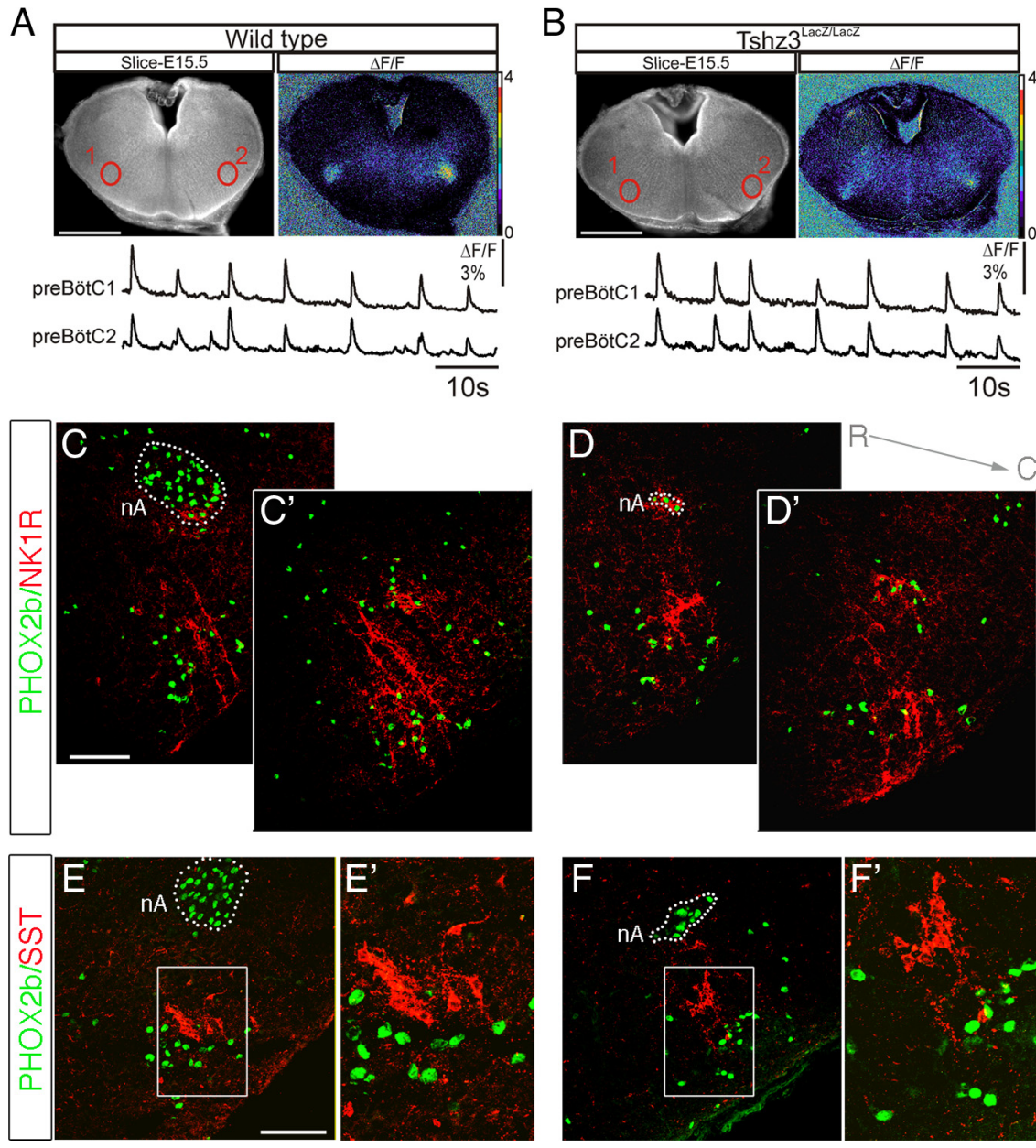

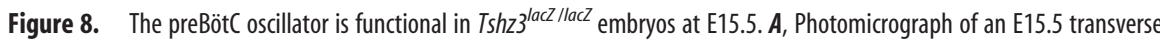
medullary slice isolating the preBötC oscillator loaded with Calcium Green-1 AM, shown in direct fluorescence (left panel) and as relative fluorescence changes ( $\Delta F / F$; right panel). The bottom traces correspond to spontaneous fluorescence changes measured preBötC oscillators (numbered 1 and 2) outlined in red in the top left panel. B, Same legend as in $\boldsymbol{A}$ for a Tshz $3^{\text {lacz } / l a c z}$ embryo. Spontaneous rhythmic activity in the preBötC region is present in preparations obtained from mutant embryos. $\mathbf{C}-\boldsymbol{F}^{\prime}$

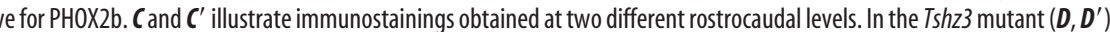

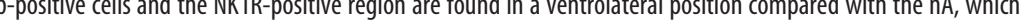
sections of WT $\left(\boldsymbol{E}, \boldsymbol{E}^{\prime}\right)$ and $T$ shzz 3 lacz /lacz $\left(\boldsymbol{F}, \boldsymbol{F}^{\prime}\right)$ embryos at E18.5. $\boldsymbol{E}^{\prime}$ and $\boldsymbol{F}^{\prime}$ correspond to the regions outlined in $\boldsymbol{E}$ and $\boldsymbol{F}$, respectively, presented at a higher magnification. The preBötC is anatomically and functionally preserved in the $T s h z 3$ mutant. Scale bars: $\boldsymbol{A}, \boldsymbol{B}, 500 \mu \mathrm{m} ; \boldsymbol{C}-\boldsymbol{D}^{\prime}, \boldsymbol{E}, \boldsymbol{F}, 100 \mu \mathrm{m}$. R, Rostral; C, caudal; SST, somatostatin.

Inactivation of $T \operatorname{sh} z 3$ alters the functional emergence of interneurons forming the e-pF, the maturation of central respiratory rhythmogenesis, and chemosensitivity

A second potential explanation for the respiratory failure in $T s h z 3^{\text {lac } / l a c z}$ mice was a dysfunction of the RGG. Indeed, at E14.5, we found that no significant rhythmically organized activity could be detected in the parafacial region in en bloc mutant preparations. It was previously shown that pharmacological inhibition or genetic elimination of the e-pF results in a slower rhythm driven by the sole preBötC oscillator (Onimaru and Homma, 2003; Dubreuil et al., 2009; Thoby-Brisson et al., 2009). This demonstrates that e-pF activity is important for scaling the frequency of fetal breathing. In addition, it has been proposed that the e-pF plays a critical role in the dynamic processes that allow the continuous generation of rhythmic motor bursts between E14.5 and E15.5 (Dubreuil et al., 2009; Thoby-Brisson et 


\section{A Wild type}
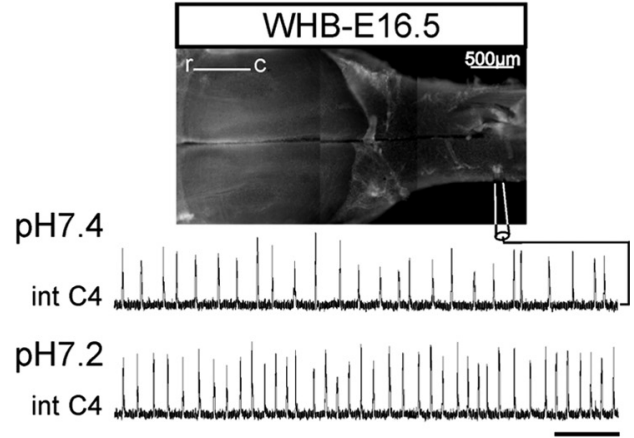

B Tshżlaczlacz

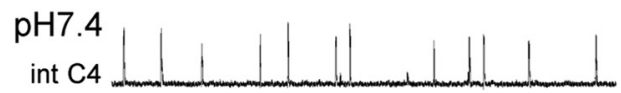

$\mathrm{pH} 7.2$

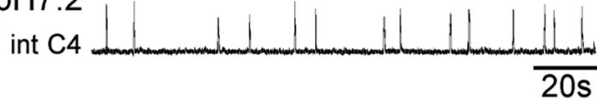

C

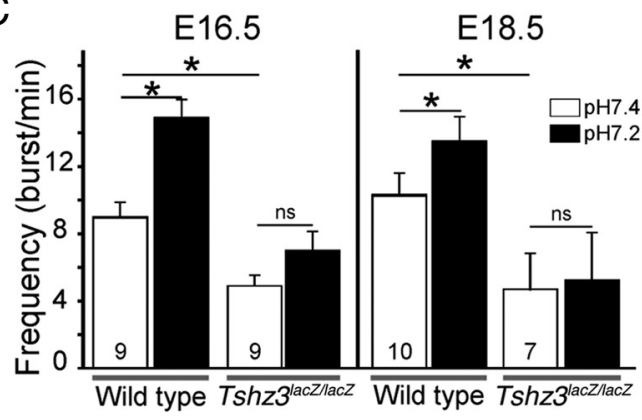

Figure 9. Slowed-down respiratory-like rhythm and lack of response to a low pH challenge in Tshz $3^{\text {lacz llacz }}$ embryos. A, Photomicrograph of a whole hindbrain preparation obtained from an E16.5 embry0, with the positioning of the recording electrode. Shown are integrated phrenic nerve discharges (Int (4) at pH 7.4 (top trace) and pH 7.2 (bottom trace) for a WT embryo. $\boldsymbol{B}$, Same legend as in $A$ for a $T s h z 3^{l a c z} / / a c z$ embryo. C, Quantification of burst frequencies for WT and

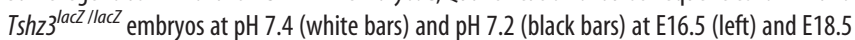
(right). The asterisks indicate significantly different values $(p<0.05)$. Numbers of hindbrain preparations analyzed are indicated on the bars. The motor output of the respiratory network recorded from ( 4 nerve roots is significantly lower in the mutants and does not respond to low pH at either E16.5 or E18.5. r, Rostral; c, caudal.

al., 2009). Consequently, the slow in vitro rhythm observed in $T s h z 3$ mutant may be attributable to a lack of entrainment of the preBötC oscillator by the excitatory drive emerging from the e-pF respiratory oscillator.

Interestingly, no anatomical defects potentially underlying the functional deficits of the e-pF could be detected in the parafacial region of $T s h z 3^{\text {lacZ/lacZ }}$ embryos. Compared with mouse lines bearing mutations in Phox2b, Lbx1, Egr2, and Atoh1 genes (Dubreuil et al., 2008, 2009; Pagliardini et al., 2008; Rose et al., 2009; Thoby-Brisson et al., 2009), the Tshz $3^{\text {lac } Z / l a c Z}$ line provides the first example of a mutation causing a functional deficit of the $\mathrm{e}-\mathrm{pF}$ that is not associated with cell loss. Whereas Phox $2 \mathrm{~b}$ or Egr2, for example, are required for e-pF neuron differentiation (Dubreuil et al., 2008, 2009), Tshz3 seems to be involved in later steps of maturation and in particular the specification of cellular properties critical for rhythm generation. This is indeed suggested by our observation that e-pF neurons of Tshz3 mutant embryos exhibit abnormal bursting properties, preventing gen- eration of rhythmically organized population activity. Additional experiments focusing on the analysis of membrane conductance involved in burst generation are now necessary for the identification of the cellular and network properties altered in the mutant. An additional nonexclusive possibility is that the functional defect of the e-pF is a non-cell-autonomous effect of the mutation.

In contrast to the e-pF, the preBötC has been found to be anatomically and functionally preserved in Tshz3 mutant embryos. By uncoupling the e-pF and preBötC oscillators, these data provide novel insights into the development and function of the RRG. First, they show that the establishment of the preBötC oscillator is independent of the neighboring nA motoneurons. Second, they confirm that timely functional emergence of the preBötC oscillator at E15.5 is not dependent on the presence of a functional e-pF. This is in accordance to what has been previously observed using other genetically modified mice in which rhythmic activity could be detected in the preBötC despite an anatomically and functionally altered e-pF (Jacquin et al., 1996; Pagliardini et al., 2008; Dubreuil et al., 2009). Third, the observation that phrenic nerve activity is generated at an abnormally slow frequency at E16.5 confirms that at developmental stages at which the e-pF and the preBötC are known to interact to form the $R R G$, the preBötC is not alone sufficient to generate an appropriate respiratory rhythm (Pagliardini et al., 2008; Dubreuil et al., 2009; Thoby-Brisson et al., 2009).

At birth or at exteriorization, after interruption of fetal umbilical irrigation by the maternal blood, central chemosensitivity is especially crucial for maintaining RRG activity and initiating air breathing movements. Tshz3 mutant embryos lacked $\mathrm{pH}$ responsiveness and failed to breathe. The retrotrapezoid nucleus $(\mathrm{RTN}) / \mathrm{pFRG}$ (the adult form of the e-pF) (Guyenet and Mulkey, 2010) neurons and $5-\mathrm{HT}^{+}$medullary neurons play an important role in central chemosensitivity. Because the e-pF oscillator is pH-sensitive at embryonic stages (Dubreuil et al., 2009) and because the e-pF is not functional in the Tshz3 mutant, it is tempting to directly associate the deficient chemosensitivity with the absence of rhythmicity in the e-pF. Such a primordial role for the $\mathrm{e}-\mathrm{pF}$ in mediating response to $\mathrm{pH}$ challenge has also been concluded from analysis of mouse lines lacking a functional e-pF (Dubreuil et al., 2008, 2009; Pagliardini et al., 2008; Rose et al., 2009; Thoby-Brisson et al., 2009). However, the role of 5-HT ${ }^{+}$ medullary neurons cannot be completely ruled out. We detected no loss of 5-HT-expressing neurons in Tshz $3^{\text {lacZ/lacZ }}$ embryos. Therefore, the possibility remains that, as presently shown for the e-pF neurons, the $T s h z 3$ mutation affects their function without affecting their anatomical presence.

Overall, our data show that a single gene, Tshz3, plays a critical role in coordinating multiple aspects of the embryonic development of the machinery required for survival at birth. It supports the functional emergence of the e-pF respiratory oscillator, which plays an important role in central chemosensitivity, and when coupled with the preBötC, in the control of the chest pump and, in parallel, is required for the survival of $\mathrm{nA}$ motoneurons that governs the upper airway valve. The functional organization of the hindbrain and its role in coordinating motor activities and breathing rhythms depend during the embryonic development on the process of segmentation. Hox and Tshz genes are critical for segment identity, and in Drosophila TSHZ acts as a cofactor of HOX proteins (Manfroid et al., 2004; Taghli-Lamallem et al., 2007). Interestingly, Tshz3 mutation affects motor activity and breathing rhythms, two activities that are also controlled by Hox genes (Chatonnet et al., 2003; Guthrie, 2007). Thus, Tshz3 may be considered to be one of the key regulators of neonatal breathing behavior. 


\section{References}

Araki K, Shiotani A, Watabe K, Saito K, Moro K, Ogawa K (2006) Adenoviral GDNF gene transfer enhances neurofunctional recovery after recurrent laryngeal nerve injury. Gene Ther 13:296-303.

Bieger D, Hopkins DA (1987) Viscerotopic representation of the upper alimentary tract in the medulla oblongata in the rat: the nucleus ambiguus. J Comp Neurol 262:546-562.

Blanchi B, Kelly LM, Viemari JC, Lafon I, Burnet H, Bévengut M, Tillmanns S, Daniel L, Graf T, Hilaire G, Sieweke MH (2003) MafB deficiency causes defective respiratory rhythmogenesis and fatal central apnea at birth. Nat Neurosci 6:1091-1100.

Caubit X, Coré N, Boned A, Kerridge S, Djabali M, Fasano L (2000) Vertebrate orthologues of the Drosophila region-specific patterning gene teashirt. Mech Dev 91:445-448.

Caubit X, Tiveron MC, Cremer H, Fasano L (2005) Expression patterns of the three Teashirt-related genes define specific boundaries in the developing and postnatal mouse forebrain. J Comp Neurol 486:76-88.

Caubit X, Lye CM, Martin E, Coré N, Long DA, Vola C, Jenkins D, Garratt AN, Skaer H, Woolf AS, Fasano L (2008) Teashirt 3 is necessary for ureteral smooth muscle differentiation downstream of SHH and BMP4. Development 135:3301-3310.

Chatonnet F, Domínguez del Toro E, Thoby-Brisson M, Champagnat J, Fortin G, Rijli FM, Thaëron-Antôno C (2003) From hindbrain segmentation to breathing after birth: developmental patterning in rhombomeres 3 and 4 . Mol Neurobiol 28:277-294.

Cheng L, Arata A, Mizuguchi R, Qian Y, Karunaratne A, Gray PA, Arata S, Shirasawa S, Bouchard M, Luo P, Chen CL, Busslinger M, Goulding M, Onimaru H, Ma Q (2004) Tlx3 and Tlx1 are post-mitotic selector genes determining glutamatergic over GABAergic cell fates. Nat Neurosci 7:510-517.

Coré N, Caubit X, Metchat A, Boned A, Djabali M, Fasano L (2007) Tshz1 is required for axial skeleton, soft palate and middle ear development in mice. Dev Biol 308:407-420.

Dauger S, Pattyn A, Lofaso F, Gaultier C, Goridis C, Gallego J, Brunet JF (2003) Phox $2 \mathrm{~b}$ controls the development of peripheral chemoreceptors and afferent visceral pathways. Development 130:6635-6642.

Dubreuil V, Ramanantsoa N, Trochet D, Vaubourg V, Amiel J, Gallego J, Brunet JF, Goridis C (2008) A human mutation in Phox 2b causes lack of $\mathrm{CO}_{2}$ chemosensitivity, fatal central apnea, and specific loss of parafacial neurons. Proc Natl Acad Sci U S A 105:1067-1072.

Dubreuil V, Thoby-Brisson M, Rallu M, Persson K, Pattyn A, Birchmeier C, Brunet JF, Fortin G, Goridis C (2009) Defective respiratory rhythmogenesis and loss of central chemosensitivity in Phox $2 \mathrm{~b}$ mutants targeting retrotrapezoid nucleus neurons. J Neurosci 29:14836-14846.

Escurat M, Djabali K, Gumpel M, Gros F, Portier MM (1990) Differential expression of two neuronal intermediate-filament proteins, peripherin and the low-molecular-mass neurofilament protein (NF-L), during the development of the rat. J Neurosci 10:764-784.

Fasano L, Röder L, Coré N, Alexandre E, Vola C, Jaca B, Kerridge S (1991) The gene teashirt is required for the development of Drosophila embryonic trunk segments and encodes a protein with widely spaced zinc finger motifs. Cell 64:63-79.

Gray PA, Rekling JC, Bocchiaro CM, Feldman JL (1999) Modulation of respiratory frequency by peptidergic input to rhythmogenic neurons in the preBotzinger complex. Science 286:1566-1568.

Guthrie S (2007) Patterning and axon guidance of cranial motor neurons. Nat Rev Neurosci 8:859-871.

Guyenet PG, Mulkey DK (2010) Retrotrapezoid nucleus and parafacial respiratory group. Respir Physiol Neurobiol. Advance online publication. Retrieved June 17, 2010. doi:10.1016/j.resp.2010.02.005.

Hirsch MR, Tiveron MC, Guillemot F, Brunet JF, Goridis C (1998) Control of noradrenergic differentiation and Phox2a expression by MASH1 in the central and peripheral nervous system. Development 125:599-608.

Jacquin TD, Borday V, Schneider-Maunoury S, Topilko P, Ghilini G, Kato F, Charnay P, Champagnat J (1996) Reorganization of pontine rhythmogenic neuronal networks in Krox-20 knock-out mice. Neuron 17: 747-758.

Jenkins D, Caubit X, Dimovski A, Matevska N, Lye CM, Cabuk F, Gucev Z, Tasic V, Fasano L, Woolf AS (2010) Analysis of TSHZ2 and TSHZ3 genes in congenital pelvi-ureteric junction obstruction. Nephrol Dial Transplant 25:54-60.

Kajiwara Y, Akram A, Katsel P, Haroutunian V, Schmeidler J, Beecham G,
Haines JL, Pericak-Vance MA, Buxbaum JD (2009) FE65 binds Teashirt, inhibiting expression of the primate-specific caspase-4. PLoS One 4:e5071.

Koebernick K, Kashef J, Pieler T, Wedlich D (2006) Xenopus Teashirt1 regulates posterior identity in brain and cranial neural crest. Dev Biol 298:312-326.

Li M, Sendtner M, Smith A (1995) Essential function of LIF receptor in motor neurons. Nature 378:724-727.

Maina F, Hilton MC, Ponzetto C, Davies AM, Klein R (1997) Met receptor signaling is required for sensory nerve development and HGF promotes axonal growth and survival of sensory neurons. Genes Dev 11:3341-3350.

Manfroid I, Caubit X, Kerridge S, Fasano L (2004) Three putative murine Teashirt orthologues specify trunk structures in Drosophila in the same way as the Drosophila teashirt gene. Development 131:1065-1073.

Nakashima K, Wiese S, Yanagisawa M, Arakawa H, Kimura N, Hisatsune T, Yoshida K, Kishimoto T, Sendtner M, Taga T (1999) Developmental requirement of gp130 signaling in neuronal survival and astrocyte differentiation. J Neurosci 19:5429-5434.

Nattie E (2006) Why do we have both peripheral and central chemoreceptors? J Appl Physiol 100:9-10.

Nattie EE, Li A, Richerson GB, Lappi DA (2004) Medullary serotonergic neurones and adjacent neurones that express neurokinin-1 receptors are both involved in chemoreception in vivo. J Physiol 556:235-253.

Onai T, Matsuo-Takasaki M, Inomata H, Aramaki T, Matsumura M, Yakura R, Sasai N, Sasai Y (2007) XTsh3 is an essential enhancing factor of canonical Wnt signaling in Xenopus axial determination. EMBO J 26:2350-2360.

Onimaru H, Homma I (2003) A novel functional neuron group for respiratory rhythm generation in the ventral medulla. J Neurosci 23:1478-1486.

Pagliardini S, Ren J, Gray PA, Vandunk C, Gross M, Goulding M, Greer JJ (2008) Central respiratory rhythmogenesis is abnormal in lbx1-deficient mice. J Neurosci 28:11030-11041.

Pattyn A, Morin X, Cremer H, Goridis C, Brunet JF (1997) Expression and interactions of the two closely related homeobox genes Phox2a and Phox2b during neurogenesis. Development 124:4065-4075.

Pattyn A, Hirsch M, Goridis C, Brunet JF (2000) Control of hindbrain motor neuron differentiation by the homeobox gene Phox $2 b$. Development 127:1349-1358.

Richerson GB (2004) Serotonergic neurons as carbon dioxide sensors that maintain $\mathrm{pH}$ homeostasis. Nat Rev Neurosci 5:449-461.

Rose MF, Ren J, Ahmad KA, Chao HT, Klisch TJ, Flora A, Greer JJ, Zoghbi HY (2009) Math1 is essential for the development of hindbrain neurons critical for perinatal breathing. Neuron 64:341-354.

Smith JC, Ellenberger HH, Ballanyi K, Richter DW, Feldman JL (1991) The Pre-Bötzinger complex: a brainstream region that may generate respiratory rhythm in mammals. Science 254:726-729.

Standish A, Enquist LW, Schwaber JS (1994) Innervation of the heart and its central medullary origin defined by viral tracing. Science 263:232-234.

Stornetta RL, Rosin DL, Wang H, Sevigny CP, Weston MC, Guyenet PG (2003) A group of glutamatergic interneurons expressing high levels of both neurokinin- 1 receptors and somatostatin identifies the region of the pre-Botzinger complex. J Comp Neurol 455:499-512.

Taghli-Lamallem O, Gallet A, Leroy F, Malapert P, Vola C, Kerridge S, Fasano L (2007) Direct interaction between Teashirt and Sex combs reduced proteins, via Tsh's acidic domain, is essential for specifying the identity of the prothorax in Drosophila. Dev Biol 307:142-151.

Thoby-Brisson M, Greer JJ (2008) Anatomical and functional development of the pre-Botzinger complex in prenatal rodents. J Appl Physiol 104:1213-1219.

Thoby-Brisson M, Trinh JB, Champagnat J, Fortin G (2005) Emergence of the pre-Botzinger respiratory rhythm generator in the mouse embryo. J Neurosci 25:4307-4318.

Thoby-Brisson M, Karlén M, Wu N, Charnay P, Champagnat J, Fortin G (2009) Genetic identification of an embryonic parafacial oscillator coupling to the preBotzinger complex. Nat Neurosci 12:1028-1035.

Tiveron MC, Hirsch MR, Brunet JF (1996) The expression pattern of the transcription factor Phox 2 delineates synaptic pathways of the autonomic nervous system. J Neurosci 16:7649-7660.

Viemari JC, Roux JC, Tryba AK, Saywell V, Burnet H, Peña F, Zanella S, Bévengut M, Barthelemy-Requin M, Herzing LB, Moncla A, Mancini J, Ramirez JM, Villard L, Hilaire G (2005) Mecp2 deficiency disrupts norepinephrine and respiratory systems in mice. J Neurosci 25:11521-11530. 